\title{
Experimental and Theoretical Studies of the Photoreduction of Copper(II)- Dendrimer Complexes
}

Haiying Wan, Shenggang Li, Tatyana A. Konovalova, Shelby Shuler, David A. Dixon,* and Shane C. Street*

Chemistry Department, The University of Alabama, Shelby Hall, Box 870336, Tuscaloosa, Alabama 35487-0336

Supporting Information Cartesian coordinates of the optimized structures and their total electronic and zero-point energies. This material is available free of charge via the Internet at http://pubs.acs.org.

Table S1. Cartesian coordinates in Angstroms for the ligands and the model $\mathrm{Cu}(\mathrm{II})$ complexes at the B3LYP/DZVP2 level. The electronic and zero-point energies in Hartree are also listed. All species containing the $\mathrm{Cu}(\mathrm{II})$ ion have a spin multiplicity of two, whereas the ligands have a spin multiplicity of one.

$$
\begin{aligned}
& \mathrm{Cu}^{2+}, \mathrm{E}_{\mathrm{e}}=-1639.1210598 \\
& \begin{array}{lrrr}
\mathrm{H}_{2} \mathrm{O}, \mathrm{E}_{\mathrm{e}}=-76.456993, \mathrm{ZPE}= & 0.0214491 & & \\
\mathrm{O} & 0.00000000 & 0.00000000 & 0.11695400 \\
\mathrm{H} & 0.00000000 & 0.76999700 & -0.46781600 \\
\mathrm{H} & 0.00000000 & -0.76999700 & -0.46781600
\end{array} \\
& \mathrm{NH}_{3}, \mathrm{E}_{\mathrm{e}}=-56.5809548, \mathrm{ZPE}=0.034544 \\
& \begin{array}{lrrr}
\mathrm{N} & 1.09865468 & 0.02242152 & 0.00000000 \\
\mathrm{H} & 1.43197657 & -0.92039156 & 0.00000000 \\
\mathrm{H} & 1.43199378 & 0.49382170 & 0.81649673 \\
\mathrm{H} & 1.43199378 & 0.49382170 & -0.81649673
\end{array} \\
& \mathrm{~N}\left(\mathrm{CH}_{3}\right)_{3}, \mathrm{E}_{\mathrm{e}}=-174.5224136, \mathrm{ZPE}=0.1202433 \\
& \begin{array}{lrrr}
\mathrm{N} & -0.77109800 & 0.00006200 & -0.00012800 \\
\mathrm{C} & -1.08737600 & -0.39476000 & 1.40001900 \\
\mathrm{H} & -0.71153800 & 0.34971700 & 2.10854700 \\
\mathrm{H} & -2.18729600 & -0.42135900 & 1.50308600 \\
\mathrm{H} & -0.71521400 & -1.40132900 & 1.61368300 \\
\mathrm{C} & -1.08723000 & -1.01529800 & -1.04188200 \\
\mathrm{H} & -2.18713500 & -1.09168900 & -1.11621300 \\
\mathrm{H} & -0.71538900 & -0.69716300 & -2.02063900 \\
\mathrm{H} & -0.71090800 & -2.00080000 & -0.75094200
\end{array}
\end{aligned}
$$




$\begin{array}{lllr}\mathrm{C} & -1.08715200 & 1.41011700 & -0.35823000 \\ \mathrm{H} & -2.18707000 & 1.51316500 & -0.38532100 \\ \mathrm{H} & -0.71371700 & 2.09855100 & 0.40598800 \\ \mathrm{H} & -0.71241400 & 1.65083500 & -1.35777200\end{array}$

$\mathrm{NH}_{2} \mathrm{CH}_{3}, \mathrm{E}_{\mathrm{e}}=-95.890914, \mathrm{ZPE}=0.0641766$

$\begin{array}{lrrr}\mathrm{N} & 0.75465700 & -0.00000500 & -0.12222700 \\ \mathrm{H} & 1.15430000 & -0.81620500 & 0.33426700 \\ \mathrm{H} & 1.15426200 & 0.81622100 & 0.33425100 \\ \mathrm{C} & -0.71148100 & -0.00000500 & 0.01766900 \\ \mathrm{H} & -1.12008400 & 0.88111000 & -0.48680900 \\ \mathrm{H} & -1.12018500 & -0.88064900 & -0.48755700 \\ \mathrm{H} & -1.08200300 & -0.00040900 & 1.05542500\end{array}$

$\mathrm{CH}_{3} \mathrm{NHCOCH}_{3}, \mathrm{E}_{\mathrm{e}}=-248.5918436, \mathrm{ZPE}=0.1019903$

$\begin{array}{lrrr}\mathrm{N} & 0.65651100 & -0.66856200 & -0.07699600 \\ \mathrm{C} & 1.90198600 & 0.07898900 & 0.03682400 \\ \mathrm{H} & 2.11414800 & 0.39976000 & 1.06548400 \\ \mathrm{H} & 2.72508700 & -0.55640700 & -0.29813700 \\ \mathrm{H} & 1.88741400 & 0.96349800 & -0.60511000 \\ \mathrm{C} & -0.61976200 & -0.16100500 & -0.00605000 \\ \mathrm{C} & -0.75493500 & 1.35533100 & -0.00912400 \\ \mathrm{H} & -0.36461700 & 1.77917000 & -0.94050300 \\ \mathrm{H} & -1.81227700 & 1.60036800 & 0.08094600 \\ \mathrm{H} & -0.20277900 & 1.80721500 & 0.82127800 \\ \mathrm{O} & -1.59977300 & -0.90951400 & 0.03573900 \\ \mathrm{H} & 0.69189800 & -1.67744600 & -0.00080400\end{array}$

$\left(\mathrm{CH}_{3}\right)_{2} \mathrm{NCH}_{2} \mathrm{CH}_{2} \mathrm{~N}\left(\mathrm{CH}_{3}\right)_{2}, \mathrm{E}_{\mathrm{e}}=-347.8360773, \mathrm{ZPE}=0.2227412$

$\begin{array}{lrrr}\mathrm{C} & -0.70821400 & 0.29782600 & 0.95356100 \\ \mathrm{C} & 0.70821300 & -0.29789600 & 0.95353900 \\ \mathrm{H} & -1.21379000 & -0.10077400 & 1.85776800 \\ \mathrm{H} & -0.64887600 & 1.38323600 & 1.09197100 \\ \mathrm{H} & 0.64886800 & -1.38331500 & 1.09186800 \\ \mathrm{H} & 1.21378800 & 0.10063400 & 1.85777800 \\ \mathrm{~N} & -1.49439700 & 0.05473100 & -0.25520700 \\ \mathrm{~N} & 1.49440300 & -0.05472500 & -0.25521400 \\ \mathrm{C} & -2.80747400 & 0.68091900 & -0.14486300 \\ \mathrm{H} & -3.35337200 & 0.56376300 & -1.08635300 \\ \mathrm{H} & -3.42876200 & 0.25258400 & 0.66667700 \\ \mathrm{H} & -2.69404500 & 1.75277100 & 0.04927600 \\ \mathrm{C} & 1.60741400 & 1.36151800 & -0.59215400 \\ \mathrm{H} & 2.07771800 & 1.96294100 & 0.21113300 \\ \mathrm{H} & 2.21899000 & 1.46835900 & -1.49337000 \\ \mathrm{H} & 0.61814600 & 1.76940400 & -0.80946200 \\ \mathrm{C} & 2.80749600 & -0.68087800 & -0.14488300 \\ \mathrm{H} & 3.35340900 & -0.56364300 & -1.08635500\end{array}$




$\begin{array}{lrrr}\mathrm{H} & 3.42875300 & -0.25257400 & 0.66669600 \\ \mathrm{H} & 2.69409600 & -1.75274700 & 0.04918300 \\ \mathrm{C} & -1.60743800 & -1.36149800 & -0.59219800 \\ \mathrm{H} & -2.21897500 & -1.46828800 & -1.49344700 \\ \mathrm{H} & -0.61817000 & -1.76940700 & -0.80946800 \\ \mathrm{H} & -2.07780300 & -1.96293200 & 0.21104400\end{array}$

$\begin{array}{crrr}\mathrm{H}_{2} \mathrm{NCH}_{2} \mathrm{CH}_{2} \mathrm{NHCOCH}_{3}, \mathrm{E}_{\mathrm{e}}=-343.2880154, \mathrm{ZPE}=0.1487427 \\ \mathrm{~N} & -0.17818100 & 0.18352600 & -0.05043300 \\ \mathrm{~N} & 2.55915300 & 0.89677000 & -0.18683800 \\ \mathrm{C} & 0.82703900 & -0.85753300 & -0.19949100 \\ \mathrm{C} & 2.15263100 & -0.39295800 & 0.40836900 \\ \mathrm{H} & 3.34053600 & 1.29828300 & 0.32454900 \\ \mathrm{H} & 2.87228300 & 0.76006800 & -1.14616500 \\ \mathrm{H} & 2.01734700 & -0.23474500 & 1.48340900 \\ \mathrm{H} & 2.89783500 & -1.19238900 & 0.28138000 \\ \mathrm{H} & 0.97051100 & -1.10938600 & -1.26124300 \\ \mathrm{H} & 0.46694700 & -1.76159400 & 0.29626600 \\ \mathrm{C} & -1.51911000 & -0.07746400 & -0.01053400 \\ \mathrm{O} & -1.97606800 & -1.22504300 & -0.04362800 \\ \mathrm{C} & -2.40963500 & 1.15576800 & 0.09591800 \\ \mathrm{H} & -2.25512800 & 1.82348300 & -0.75824300 \\ \mathrm{H} & -3.45016200 & 0.83358200 & 0.11665100 \\ \mathrm{H} & -2.18606300 & 1.71792600 & 1.00853900 \\ \mathrm{H} & 0.16208400 & 1.13616300 & -0.10079200\end{array}$

$\mathrm{CH}_{3} \mathrm{NHCOCH}_{2} \mathrm{CH}_{2} \mathrm{~N}\left(\mathrm{CH}_{3}\right) \mathrm{CH}_{2} \mathrm{CH}_{2} \mathrm{~N}\left(\mathrm{CH}_{3}\right) \mathrm{CH}_{2} \mathrm{CH}_{2} \mathrm{CONHCH}_{3}, \mathrm{E}_{\mathrm{e}}=-842.6051827, \mathrm{ZPE}$ $=0.3906328$

$\begin{array}{lrrr}\mathrm{C} & 0.40004200 & -1.68391800 & -0.71863800 \\ \mathrm{H} & -0.09202200 & -0.93093200 & -1.35956400 \\ \mathrm{H} & 0.08539100 & -2.66074300 & -1.09567000 \\ \mathrm{C} & -0.09340100 & -1.58082700 & 0.73433200 \\ \mathrm{H} & 0.27413900 & -0.65393800 & 1.21239200 \\ \mathrm{H} & 0.35538300 & -2.41104800 & 1.28652700 \\ \mathrm{~N} & -1.55512500 & -1.69356000 & 0.83658700 \\ \mathrm{~N} & 1.86473800 & -1.59782500 & -0.81694800 \\ \mathrm{C} & -1.95695300 & -2.19036800 & 2.15250600 \\ \mathrm{H} & -1.69041700 & -1.49826400 & 2.97494700 \\ \mathrm{H} & -3.03677500 & -2.35422200 & 2.18544300 \\ \mathrm{C} & -2.22726700 & -0.43356500 & 0.50194700 \\ \mathrm{H} & -2.10284100 & 0.29622200 & 1.32984900 \\ \mathrm{H} & -1.73172500 & -0.00522500 & -0.37284600 \\ \mathrm{C} & -3.71650000 & -0.62905100 & 0.16935900 \\ \mathrm{H} & -3.81909300 & -1.42618500 & -0.56915800 \\ \mathrm{H} & -4.27740600 & -0.94494500 & 1.05613500 \\ \mathrm{C} & -4.45858200 & 0.58118100 & -0.40156300 \\ \mathrm{O} & -5.39057100 & 0.43881200 & -1.19779400\end{array}$




$\begin{array}{lrrr}\mathrm{N} & -4.05094000 & 1.81405000 & 0.04208200 \\ \mathrm{H} & -3.29782400 & 1.86053500 & 0.71226700 \\ \mathrm{C} & -4.69549100 & 3.05304100 & -0.37421800 \\ \mathrm{H} & -3.96432900 & 3.75024200 & -0.79668900 \\ \mathrm{H} & -5.43270400 & 2.80206300 & -1.13679200 \\ \mathrm{C} & 2.34516700 & -2.27278300 & -2.02545100 \\ \mathrm{H} & 1.96874700 & -1.80880400 & -2.95587000 \\ \mathrm{H} & 3.43718400 & -2.25936300 & -2.06110100 \\ \mathrm{C} & 2.33209900 & -0.20659700 & -0.75599400 \\ \mathrm{H} & 2.15163300 & 0.32925600 & -1.70404000 \\ \mathrm{H} & 1.75670600 & 0.31232600 & 0.01625500 \\ \mathrm{C} & 3.83030800 & -0.09737700 & -0.40279900 \\ \mathrm{H} & 4.44120100 & -0.51909900 & -1.20353000 \\ \mathrm{H} & 4.02380500 & -0.66404400 & 0.51072700 \\ \mathrm{C} & 4.23723600 & 1.36700700 & -0.28732600 \\ \mathrm{O} & 4.41881800 & 2.06504600 & -1.29064700 \\ \mathrm{~N} & 4.34956000 & 1.90564000 & 0.97205800 \\ \mathrm{H} & 4.65464600 & 2.87091200 & 0.96679700 \\ \mathrm{C} & 4.28308000 & 1.20918800 & 2.25206900 \\ \mathrm{H} & 5.12702800 & 0.52535100 & 2.40460700 \\ \mathrm{H} & 3.35253000 & 0.64361500 & 2.35498400 \\ \mathrm{H} & 2.02886500 & -3.31944200 & -2.01013900 \\ \mathrm{H} & -1.47295200 & -3.15163100 & 2.34474000 \\ \mathrm{H} & -5.20630100 & 3.53830000 & 0.46541000 \\ \mathrm{H} & 4.30509700 & 1.95448500 & 3.05004200 \\ & & & \\ & & & \\ & & & \end{array}$

\section{$\left(\mathrm{CH}_{3} \mathrm{NHCOCH}_{2} \mathrm{CH}_{2}\right)_{2} \mathrm{NCH}_{2} \mathrm{CH}_{2} \mathrm{~N}\left(\mathrm{CH}_{2} \mathrm{CH}_{2} \mathrm{CONHCH}_{3}\right)_{2}$,}

\section{$\mathrm{E}_{\mathrm{e}}=-1337.3890727, \mathrm{ZPE}=0.5586884$}

$\begin{array}{lr}\mathrm{C} & -0.54797200 \\ \mathrm{H} & -0.32936700 \\ \mathrm{H} & -0.43755700 \\ \mathrm{C} & 0.48783700 \\ \mathrm{H} & 0.32092300 \\ \mathrm{H} & 0.31468100 \\ \mathrm{~N} & 1.86732300 \\ \mathrm{~N} & -1.92290600 \\ \mathrm{C} & 2.78560600 \\ \mathrm{H} & 2.62190000 \\ \mathrm{H} & 3.80884700 \\ \mathrm{C} & 2.33246500 \\ \mathrm{H} & 2.68335200 \\ \mathrm{H} & 1.49670200 \\ \mathrm{C} & 2.68877800 \\ \mathrm{H} & 1.66532500 \\ \mathrm{H} & 2.91167300 \\ \mathrm{C} & 3.43327800 \\ \mathrm{H} & 3.06723300 \\ & \end{array}$

$$
\begin{array}{rr}
0.04581800 & -0.59542900 \\
-0.76265200 & -1.31281800 \\
0.98019600 & -1.15158700 \\
0.05644600 & 0.53956000 \\
-0.79415900 & 1.22342100 \\
0.95692500 & 1.13428700 \\
0.06636600 & 0.02911300 \\
-0.04774400 & -0.08079200 \\
0.76895300 & 0.93706600 \\
0.49976900 & 1.99177200 \\
0.46645000 & 0.69562500 \\
-1.29308400 & -0.27927900 \\
-1.81672900 & 0.62829000 \\
-1.87627900 & -0.66806600 \\
2.29570600 & 0.78658100 \\
2.62796900 & 0.99456800 \\
2.57447300 & -0.24849700 \\
-1.31536700 & -1.34988300 \\
-0.77917200 & -2.23284800
\end{array}
$$




\begin{tabular}{|c|c|c|c|}
\hline $\mathrm{H}$ & 4.33695700 & -0.79843500 & -1.00916500 \\
\hline $\mathrm{C}$ & 3.59754300 & 3.02343700 & 1.77482800 \\
\hline 0 & 3.69245600 & 2.68048900 & 2.95767300 \\
\hline $\mathrm{N}$ & 4.28750200 & 4.08910600 & 1.25489500 \\
\hline $\mathrm{H}$ & 4.13820500 & 4.32222500 & 0.28406400 \\
\hline $\mathrm{C}$ & 5.14646200 & 4.94446300 & 2.06299900 \\
\hline $\mathrm{H}$ & 6.15256100 & 5.00336400 & 1.63469900 \\
\hline $\mathrm{H}$ & 5.20685100 & 4.50382000 & 3.05834800 \\
\hline $\mathrm{C}$ & 3.76039100 & -2.74478700 & -1.78320400 \\
\hline 0 & 2.88375600 & -3.60172600 & -1.92970800 \\
\hline $\mathrm{N}$ & 5.08779100 & -2.99507600 & -2.01983200 \\
\hline $\mathrm{H}$ & 5.74580900 & -2.24230900 & -1.88114700 \\
\hline $\mathrm{C}$ & 5.57593300 & -4.27878300 & -2.50651100 \\
\hline $\mathrm{H}$ & 6.29828400 & -4.71526500 & -1.80831400 \\
\hline $\mathrm{H}$ & 4.71755900 & -4.94522300 & -2.59294000 \\
\hline $\mathrm{C}$ & -2.88788200 & 0.63824300 & -0.95294400 \\
\hline $\mathrm{H}$ & -2.67596200 & 0.46862300 & -2.01949700 \\
\hline $\mathrm{H}$ & -3.88256900 & 0.22447200 & -0.76183300 \\
\hline $\mathrm{C}$ & -2.32112600 & -1.43802200 & 0.18835400 \\
\hline $\mathrm{H}$ & -2.74054800 & -1.92909200 & -0.70304700 \\
\hline $\mathrm{H}$ & -1.42880500 & -2.00491500 & 0.46911800 \\
\hline $\mathrm{C}$ & -3.34097800 & -1.53036000 & 1.34044100 \\
\hline $\mathrm{H}$ & -4.21153000 & -0.90689000 & 1.11535400 \\
\hline $\mathrm{H}$ & -2.88436500 & -1.14403600 & 2.25747600 \\
\hline $\mathrm{C}$ & -2.92837100 & 2.15486500 & -0.68358600 \\
\hline $\mathrm{H}$ & -3.24556700 & 2.33020200 & 0.34961400 \\
\hline $\mathrm{H}$ & -1.92529700 & 2.57744100 & -0.79758200 \\
\hline $\mathrm{C}$ & -3.85559400 & -2.95715300 & 1.50832300 \\
\hline 0 & -4.53237500 & -3.50942000 & 0.63537100 \\
\hline $\mathrm{N}$ & -3.50155000 & -3.57848900 & 2.67893300 \\
\hline $\mathrm{H}$ & -2.94712200 & -3.05981600 & 3.34401100 \\
\hline $\mathrm{C}$ & -3.88970800 & -4.94638200 & 2.99617400 \\
\hline $\mathrm{H}$ & -3.00987900 & -5.58405600 & 3.13498300 \\
\hline $\mathrm{H}$ & -4.47354300 & -5.32476300 & 2.15677800 \\
\hline $\mathrm{C}$ & -3.82356100 & 2.87180700 & -1.69021800 \\
\hline 0 & -3.56485200 & 2.88225700 & -2.89783300 \\
\hline $\mathrm{N}$ & -4.92963700 & 3.48803300 & -1.16208000 \\
\hline $\mathrm{H}$ & -5.07404400 & 3.43247700 & -0.16472200 \\
\hline $\mathrm{C}$ & -5.90098300 & 4.20373300 & -1.97846700 \\
\hline $\mathrm{H}$ & -6.89173800 & 3.74107200 & -1.91082800 \\
\hline $\mathrm{H}$ & -5.55809200 & 4.15736600 & -3.01235200 \\
\hline $\mathrm{H}$ & -5.97591600 & 5.25300500 & -1.67270700 \\
\hline $\mathrm{H}$ & -4.50291300 & -4.98259700 & 3.90332900 \\
\hline $\mathrm{H}$ & 4.73578600 & 5.95694300 & 2.14755400 \\
\hline $\mathrm{H}$ & 6.04792100 & -4.17578600 & -3.48985900 \\
\hline
\end{tabular}

$\mathrm{Cu}\left(\mathrm{NH}_{2} \mathrm{CH}_{3}\right)^{2+}(\mathbf{1}), \mathrm{E}_{\mathrm{e}}=-1735.318687, \mathrm{ZPE}=0.0642355$ 


$\begin{array}{lrrr}\mathrm{N} & 0.92090400 & 0.65403700 & 0.00000100 \\ \mathrm{H} & 0.98922600 & 1.25154300 & 0.84111600 \\ \mathrm{H} & 0.98908400 & 1.25144500 & -0.84123200 \\ \mathrm{C} & 1.89606000 & -0.44597100 & -0.00001500 \\ \mathrm{H} & 1.85308800 & -1.03247700 & 0.92606500 \\ \mathrm{H} & 1.85288000 & -1.03264600 & -0.92594200 \\ \mathrm{H} & 2.92425000 & 0.01960200 & -0.00005100 \\ \mathrm{Cu} & -0.91142100 & -0.08137600 & 0.00000400\end{array}$

$\mathrm{Cu}\left[\mathrm{N}\left(\mathrm{CH}_{3}\right)_{3}\right]^{2+}(2), \mathrm{E}_{\mathrm{e}}=-1814.0033055, \mathrm{ZPE}=0.1190665$

$\begin{array}{rrrr}\mathrm{Cu} & 1.23479000 & -0.00002500 & 0.00003600 \\ \mathrm{~N} & -0.77109800 & 0.00006200 & -0.00012800 \\ \mathrm{C} & -1.08737600 & -0.39476000 & 1.40001900 \\ \mathrm{H} & -0.71153800 & 0.34971700 & 2.10854700 \\ \mathrm{H} & -2.18729600 & -0.42135900 & 1.50308600 \\ \mathrm{H} & -0.71521400 & -1.40132900 & 1.61368300 \\ \mathrm{C} & -1.08723000 & -1.01529800 & -1.04188200 \\ \mathrm{H} & -2.18713500 & -1.09168900 & -1.11621300 \\ \mathrm{H} & -0.71538900 & -0.69716300 & -2.02063900 \\ \mathrm{H} & -0.71090800 & -2.00080000 & -0.75094200 \\ \mathrm{C} & -1.08715200 & 1.41011700 & -0.35823000 \\ \mathrm{H} & -2.18707000 & 1.51316500 & -0.38532100 \\ \mathrm{H} & -0.71371700 & 2.09855100 & 0.40598800 \\ \mathrm{H} & -0.71241400 & 1.65083500 & -1.35777200\end{array}$

$\mathrm{Cu}\left[N-\mathrm{CH}_{3} \mathrm{NHCOCH}_{3}\right]^{2+}(\mathbf{3 a}), \mathrm{E}_{\mathrm{e}}=-1888.0308473, \mathrm{ZPE}=0.1009012$

$\begin{array}{lrrr}\mathrm{Cu} & -1.56017400 & -0.36580200 & 0.09954800 \\ \mathrm{~N} & 0.18312200 & 0.48809500 & -0.48870400 \\ \mathrm{C} & 0.24806400 & 1.94157400 & -0.02132600 \\ \mathrm{H} & 1.11356800 & 2.44109200 & -0.46822700 \\ \mathrm{H} & -0.65803700 & 2.43118900 & -0.39381200 \\ \mathrm{H} & 0.29368300 & 1.98097200 & 1.06656300 \\ \mathrm{C} & 1.38962100 & -0.29366600 & -0.15943900 \\ \mathrm{C} & 1.96631100 & -0.35947800 & 1.22194100 \\ \mathrm{H} & 1.17427700 & -0.30173700 & 1.97595800 \\ \mathrm{H} & 2.55545000 & -1.27376800 & 1.33305600 \\ \mathrm{H} & 2.64821700 & 0.49847800 & 1.33338000 \\ \mathrm{O} & 1.88751600 & -0.85047600 & -1.13002800 \\ \mathrm{H} & 0.11191500 & 0.48859200 & -1.51970300\end{array}$

$\mathrm{Cu}\left[O-\mathrm{CH}_{3} \mathrm{NHCOCH}_{3}\right]^{2+}(\mathbf{3 b}), \mathrm{E}_{\mathrm{e}}=-1888.0871332, \mathrm{ZPE}=0.1003309$

$\begin{array}{lrrr}\mathrm{N} & -1.95003800 & -0.73057000 & -0.00009200 \\ \mathrm{C} & -3.36155900 & -0.49130500 & 0.00085600 \\ \mathrm{H} & -3.80836700 & -1.01658600 & -0.86851300 \\ \mathrm{H} & -3.80702300 & -1.01581100 & 0.87142200 \\ \mathrm{H} & -3.61315000 & 0.56601400 & 0.00066000 \\ \mathrm{C} & -0.91027900 & 0.20469400 & -0.00068200\end{array}$




$\begin{array}{lrrr}\mathrm{C} & -1.19325700 & 1.66488000 & 0.00000100 \\ \mathrm{H} & -1.77871700 & 1.94352800 & 0.88556700 \\ \mathrm{H} & -0.26001900 & 2.22774600 & 0.00075400 \\ \mathrm{H} & -1.77745500 & 1.94418100 & -0.88625000 \\ \mathrm{O} & 0.22860400 & -0.33485500 & -0.00205100 \\ \mathrm{H} & -1.63080000 & -1.70917400 & -0.00061700 \\ \mathrm{Cu} & 2.11336300 & -0.11781600 & 0.00044800\end{array}$

$\begin{array}{crrr}\mathrm{Cu}\left(\mathrm{NH}_{2} \mathrm{CH}_{3}\right)_{2}{ }^{2+}(\mathbf{4}), \mathrm{E}_{\mathrm{e}}=-1831.3710379, \mathrm{ZPE}=0.1343296 \\ \mathrm{~N} & -1.81190100 & -0.67681200 & -0.02707100 \\ \mathrm{H} & -1.95388200 & -1.32951100 & 0.75362300 \\ \mathrm{H} & -1.99674200 & -1.21767700 & -0.88092700 \\ \mathrm{C} & -2.79585200 & 0.46135200 & 0.07634100 \\ \mathrm{H} & -2.64664000 & 0.99747700 & 1.01711300 \\ \mathrm{H} & -2.69155100 & 1.12295600 & -0.78757900 \\ \mathrm{H} & -3.81900800 & 0.05753400 & 0.07358700 \\ \mathrm{Cu} & -0.00005600 & -0.00026300 & -0.03062900 \\ \mathrm{~N} & 1.81165200 & 0.67685900 & -0.02715200 \\ \mathrm{H} & 1.99604000 & 1.21780100 & -0.88106700 \\ \mathrm{C} & 2.79630300 & -0.46062100 & 0.07635800 \\ \mathrm{H} & 2.69258600 & -1.12228700 & -0.78757700 \\ \mathrm{H} & 3.81920500 & -0.05614500 & 0.07382000 \\ \mathrm{H} & 2.64730600 & -0.99688800 & 1.01709000 \\ \mathrm{H} & 1.95334200 & 1.32964500 & 0.75352000\end{array}$

$\begin{array}{crrr}\mathrm{Cu}\left[N, N-\left(\mathrm{CH}_{3}\right)_{2} \mathrm{NCH}_{2} \mathrm{CH}_{2} \mathrm{~N}\left(\mathrm{CH}_{3}\right)_{2}\right]^{2+}(\mathbf{5}), \mathrm{E}_{\mathrm{e}}=-1987.44986, \mathrm{ZPE}=0.2256575 \\ \mathrm{C} & -0.68762500 & 1.51022200 & -0.33351800 \\ \mathrm{C} & 0.68718800 & 1.50967800 & 0.33618700 \\ \mathrm{H} & -1.26938200 & 2.37668200 & -0.00078600 \\ \mathrm{H} & -0.60246700 & 1.56780700 & -1.41995200 \\ \mathrm{H} & 0.60208700 & 1.56513900 & 1.42273200 \\ \mathrm{H} & 1.26882500 & 2.37683200 & 0.00507900 \\ \mathrm{Cu} & 0.00031100 & -1.11535400 & -0.00082900 \\ \mathrm{~N} & -1.44788000 & 0.24635300 & -0.00332300 \\ \mathrm{~N} & 1.44758000 & 0.24649200 & 0.00347300 \\ \mathrm{C} & -2.46598400 & -0.09235200 & -1.04885100 \\ \mathrm{H} & -2.97548600 & -1.02090300 & -0.78170600 \\ \mathrm{H} & -3.21273300 & 0.71053000 & -1.09502700 \\ \mathrm{H} & -1.99025100 & -0.18612700 & -2.02715600 \\ \mathrm{C} & 2.07219000 & 0.27544900 & -1.36100400 \\ \mathrm{H} & 2.85917800 & 1.03873300 & -1.37075000 \\ \mathrm{H} & 2.52624400 & -0.69318600 & -1.58036900 \\ \mathrm{H} & 1.33238300 & 0.52159500 & -2.12376500 \\ \mathrm{C} & 2.46561800 & -0.09423100 & 1.04850400 \\ \mathrm{H} & 2.97539000 & -1.02207400 & 0.77941800 \\ \mathrm{H} & 3.21217600 & 0.70871200 & 1.09662100 \\ \mathrm{H} & 1.98971500 & -0.19023000 & 2.02652200\end{array}$




$\begin{array}{lrrr}\mathrm{C} & -2.07220200 & 0.27228800 & 1.36134800 \\ \mathrm{H} & -2.52654300 & -0.69671400 & 1.57847400 \\ \mathrm{H} & -1.33220700 & 0.51644000 & 2.12456800 \\ \mathrm{H} & -2.85896900 & 1.03578900 & 1.37309200\end{array}$

\section{$\mathrm{Cu}\left[\mathrm{N}, \mathrm{O}-\mathrm{CH}_{3} \mathrm{NHCOCH}_{3}\right]^{2+}(\mathbf{6}), \mathrm{E}_{\mathrm{e}}=-1888.0581959, \mathrm{ZPE}=0.1019627$}

$\begin{array}{lrrr}\mathrm{C} & 1.02014300 & -0.52359700 & 0.04857100 \\ \mathrm{Cu} & -1.40666000 & -0.13549800 & 0.00865400 \\ \mathrm{~N} & 0.38762700 & 0.74734300 & 0.44617300 \\ \mathrm{O} & 0.10572100 & -1.31369300 & -0.28305300 \\ \mathrm{C} & 2.45601700 & -0.81141100 & 0.03940700 \\ \mathrm{H} & 2.90594900 & -0.53352400 & 1.00468300 \\ \mathrm{H} & 2.62913400 & -1.86694300 & -0.18599800 \\ \mathrm{H} & 2.94880300 & -0.18794500 & -0.72551200 \\ \mathrm{C} & 0.79234200 & 2.00429700 & -0.30111300 \\ \mathrm{H} & 0.71073600 & 1.83917300 & -1.37760000 \\ \mathrm{H} & 0.13576500 & 2.81426400 & 0.01952100 \\ \mathrm{H} & 1.82878800 & 2.24384700 & -0.03259500 \\ \mathrm{H} & 0.46378900 & 0.88298000 & 1.46657700\end{array}$

\section{$\mathrm{Cu}\left(\mathrm{H}_{2} \mathrm{O}\right)_{6}{ }^{2+}(7), \mathrm{E}_{\mathrm{e}}=-2098.4498266, \mathrm{ZPE}=0.1487003$}

$\begin{array}{lrrr}\mathrm{Cu} & -0.00260800 & 0.00205700 & 0.00433600 \\ \mathrm{O} & 0.20050000 & -1.69714900 & 1.11845400 \\ \mathrm{H} & 0.05780700 & -1.80467000 & 2.07374200 \\ \mathrm{O} & -0.07329600 & 1.20110200 & 1.62219800 \\ \mathrm{H} & 0.70657900 & 1.37504700 & 2.17846500 \\ \mathrm{O} & 2.27277700 & 0.14705200 & -0.04353800 \\ \mathrm{H} & 2.82856200 & 0.83935200 & -0.43853000 \\ \mathrm{O} & -0.15417000 & 1.70731700 & -1.10881700 \\ \mathrm{H} & -0.16005200 & 1.81670000 & -2.07402700 \\ \mathrm{O} & -2.28667000 & -0.16680200 & 0.01612800 \\ \mathrm{H} & -2.84640300 & -0.86777200 & 0.38990100 \\ \mathrm{O} & 0.06927100 & -1.19736800 & -1.61593800 \\ \mathrm{H} & 0.87448000 & -1.28377600 & -2.15651000 \\ \mathrm{H} & -0.87297100 & 1.28096300 & 2.17145600 \\ \mathrm{H} & 0.16184800 & -2.57729400 & 0.70595800 \\ \mathrm{H} & 2.88769100 & -0.48465400 & 0.36556500 \\ \mathrm{H} & -0.18259900 & 2.58896700 & -0.69973200 \\ \mathrm{H} & -0.70533100 & -1.37448400 & -2.17813500 \\ \mathrm{H} & -2.90127000 & 0.47875500 & -0.37181200\end{array}$

$\begin{array}{crrr}\mathrm{Cu}\left(\mathrm{H}_{2} \mathrm{O}\right)_{5}\left(\mathrm{NH}_{3}\right)^{2+}(\mathbf{8}), \mathrm{E}_{\mathrm{e}}=-1999.1524138, \mathrm{ZPE}=0.2179603 & \\ \mathrm{Cu} & -0.21336900 & 0.00211600 & 0.00469800 \\ \mathrm{~N} & 0.16692600 & -1.63563200 & -1.27313700 \\ \mathrm{H} & 1.07361400 & -2.04488200 & -1.03826000 \\ \mathrm{H} & -0.52428600 & -2.38402100 & -1.19450600 \\ \mathrm{~N} & 0.02872200 & 1.33132600 & -1.59842500\end{array}$




$\begin{array}{lrrr}\mathrm{H} & -0.43533900 & 2.23157900 & -1.46570700 \\ \mathrm{H} & 1.02446800 & 1.53254200 & -1.71673100 \\ \mathrm{~N} & 0.03940300 & 1.66355200 & 1.28661100 \\ \mathrm{H} & 0.93264100 & 2.11917700 & 1.08772500 \\ \mathrm{H} & -0.68391200 & 2.37597000 & 1.17160300 \\ \mathrm{~N} & -2.54992900 & -0.07521800 & -0.03612000 \\ \mathrm{H} & -2.94456900 & -0.05726700 & -0.97874600 \\ \mathrm{H} & -2.95045800 & -0.91092700 & 0.39474300 \\ \mathrm{O} & 2.77792800 & 0.02557200 & -0.00223700 \\ \mathrm{H} & 3.40025600 & -0.13867900 & -0.73006200 \\ \mathrm{H} & 3.36410500 & 0.21990700 & 0.74770200 \\ \mathrm{H} & 0.05320000 & 1.45123500 & 2.28524100 \\ \mathrm{H} & -0.30810500 & 0.98552200 & -2.49832900 \\ \mathrm{H} & -3.00378000 & 0.70188400 & 0.44826000 \\ \mathrm{H} & 0.21423200 & -1.41298900 & -2.26845000 \\ \mathrm{~N} & 0.07411300 & -1.31656600 & 1.61035700 \\ \mathrm{H} & -0.46868800 & -2.17863500 & 1.53432800 \\ \mathrm{H} & 1.05813900 & -1.59238200 & 1.64720400 \\ \mathrm{H} & -0.15187800 & -0.93621000 & 2.53063800\end{array}$

$\begin{array}{crrr}\text { trans }-\mathrm{Cu}\left(\mathrm{H}_{2} \mathrm{O}\right)_{4}\left(\mathrm{NH}_{3}\right)_{2}{ }^{2+}(\mathbf{9 a}), \mathrm{E}_{\mathrm{e}}=-2058.7421103, \mathrm{ZPE}=0.1770392 & \\ \mathrm{Cu} & 0.00324000 & -0.00131400 & 0.00048700 \\ \mathrm{O} & 0.05418400 & 2.18258600 & 0.04728200 \\ \mathrm{H} & 0.82869700 & 2.72978600 & 0.26038600 \\ \mathrm{O} & -0.10838600 & -2.18170800 & -0.04066700 \\ \mathrm{H} & -0.92522000 & -2.67355600 & -0.22975700 \\ \mathrm{O} & -2.35665500 & 0.07524100 & -0.00413300 \\ \mathrm{H} & -2.96464900 & -0.35647700 & 0.61933600 \\ \mathrm{O} & 2.36631700 & -0.07013200 & -0.00316200 \\ \mathrm{H} & 2.97406000 & 0.47600300 & -0.52958400 \\ \mathrm{~N} & 0.01659100 & -0.06814600 & 2.00204300 \\ \mathrm{H} & -0.05473800 & -1.02613100 & 2.35182100 \\ \mathrm{H} & 0.87909000 & 0.32917000 & 2.37988400 \\ \mathrm{~N} & 0.02279900 & 0.06455000 & -2.00133000 \\ \mathrm{H} & -0.80132300 & -0.37849000 & -2.41192800 \\ \mathrm{H} & 0.05821200 & 1.02488700 & -2.35017400 \\ \mathrm{H} & 0.83658400 & -0.42546000 & -2.37836300 \\ \mathrm{H} & -0.75389300 & 0.46434800 & 2.41054300 \\ \mathrm{H} & -2.93754300 & 0.55526700 & -0.61803700 \\ \mathrm{H} & 0.62627900 & -2.79203600 & -0.22117400 \\ \mathrm{H} & 2.94706100 & -0.65587500 & 0.51104600 \\ \mathrm{H} & -0.72598500 & 2.74393900 & 0.19232600\end{array}$

$\begin{array}{cccr}\text { cis }-\mathrm{Cu}\left(\mathrm{H}_{2} \mathrm{O}\right)_{4}\left(\mathrm{NH}_{3}\right)_{2}{ }^{2+}(\mathbf{9 b}), \mathrm{E}_{\mathrm{e}}=-2058.7421103, \mathrm{ZPE}=0.1770392 & \\ \mathrm{Cu} & 0.02232200 & 0.11286400 & 0.04003500 \\ \mathrm{~N} & 0.06767700 & 1.78249800 & -1.13048500 \\ \mathrm{H} & 0.91873600 & 2.33634000 & -1.01249300\end{array}$




$\begin{array}{rrrr}\mathrm{H} & 0.02288700 & 1.52543200 & -2.11875900 \\ \mathrm{~N} & 0.20459800 & 1.13236300 & 1.78826000 \\ \mathrm{H} & -0.55611800 & 0.89251000 & 2.42842600 \\ \mathrm{H} & 1.08558300 & 0.89250400 & 2.24938000 \\ \mathrm{O} & -2.42399900 & -0.02915400 & -0.03356900 \\ \mathrm{H} & -3.14345000 & 0.56030000 & -0.31483700 \\ \mathrm{O} & -0.08851100 & -1.68803100 & 1.10729900 \\ \mathrm{H} & -0.15535500 & -2.50759800 & 0.58897800 \\ \mathrm{O} & 2.38563800 & -0.32888200 & -0.25388700 \\ \mathrm{H} & 2.80556500 & -1.12267400 & 0.11640400 \\ \mathrm{O} & -0.21449000 & -1.16051000 & -1.58057200 \\ \mathrm{H} & 0.48514900 & -1.40990900 & -2.20795600 \\ \mathrm{H} & 0.06710400 & -1.93827100 & 2.03268200 \\ \mathrm{H} & -2.86538800 & -0.72834300 & 0.47649200 \\ \mathrm{H} & -0.72031200 & 2.41054600 & -0.95795300 \\ \mathrm{H} & -1.07785800 & -1.25995500 & -2.01744500 \\ \mathrm{H} & 3.11826700 & 0.17438200 & -0.64634000 \\ \mathrm{H} & 0.19283300 & 2.15025900 & 1.70380600\end{array}$

$\begin{array}{lrrr}\text { trans }-\mathrm{Cu}\left(\mathrm{H}_{2} \mathrm{O}\right)_{2}\left(\mathrm{NH}_{3}\right)_{4}{ }^{2+}(\mathbf{1 0}), \mathrm{E}_{\mathrm{e}}=-2019.022993, \mathrm{ZPE}=0.2043254 & \\ \mathrm{Cu} & -0.00194300 & 0.00275200 & 0.00131100 \\ \mathrm{O} & -2.63599200 & -0.03346600 & 0.02480800 \\ \mathrm{H} & -3.24073300 & 0.67942000 & 0.28844200 \\ \mathrm{O} & 2.64113600 & 0.01730900 & -0.03051600 \\ \mathrm{H} & 3.23689900 & -0.56637500 & -0.52827700 \\ \mathrm{H} & 3.24624900 & 0.62296600 & 0.42821800 \\ \mathrm{H} & -3.23213600 & -0.76668500 & -0.19996200 \\ \mathrm{~N} & 0.00446400 & 2.05967100 & -0.29471900 \\ \mathrm{H} & 0.83711200 & 2.33160800 & -0.82221200 \\ \mathrm{H} & -0.80545200 & 2.36188100 & -0.84003900 \\ \mathrm{~N} & 0.08153100 & -2.05401700 & 0.29468600 \\ \mathrm{H} & 0.94288900 & -2.29271400 & 0.79119400 \\ \mathrm{H} & -0.69611600 & -2.38685000 & 0.86826600 \\ \mathrm{H} & 0.00474400 & 2.63886600 & 0.54651100 \\ \mathrm{H} & 0.07389400 & -2.63223800 & -0.54710500 \\ \mathrm{~N} & -0.07657700 & -0.27489900 & -2.06335600 \\ \mathrm{H} & -0.09051000 & 0.58705300 & -2.61084700 \\ \mathrm{H} & 0.71626200 & -0.81245400 & -2.41833200 \\ \mathrm{~N} & -0.00911900 & 0.27702300 & 2.06538600 \\ \mathrm{H} & 0.00177900 & -0.58530600 & 2.61239100 \\ \mathrm{H} & 0.79325300 & 0.81884200 & 2.39131800 \\ \mathrm{H} & -0.92500500 & -0.78057700 & -2.32564700 \\ \mathrm{H} & -0.85003100 & 0.77756900 & 2.35975100\end{array}$

$\mathrm{Cu}\left(\mathrm{H}_{2} \mathrm{O}\right)\left(\mathrm{NH}_{3}\right)_{5}{ }^{2+}(\mathbf{1 1}), \mathrm{E}_{\mathrm{e}}=-1999.1524138, \mathrm{ZPE}=0.2179603$

$\begin{array}{lrrr}\mathrm{Cu} & -0.21336900 & 0.00211600 & 0.00469800 \\ \mathrm{~N} & 0.16692600 & -1.63563200 & -1.27313700\end{array}$




$\begin{array}{lrrr}\mathrm{H} & 1.07361400 & -2.04488200 & -1.03826000 \\ \mathrm{H} & -0.52428600 & -2.38402100 & -1.19450600 \\ \mathrm{~N} & 0.02872200 & 1.33132600 & -1.59842500 \\ \mathrm{H} & -0.43533900 & 2.23157900 & -1.46570700 \\ \mathrm{H} & 1.02446800 & 1.53254200 & -1.71673100 \\ \mathrm{~N} & 0.03940300 & 1.66355200 & 1.28661100 \\ \mathrm{H} & 0.93264100 & 2.11917700 & 1.08772500 \\ \mathrm{H} & -0.68391200 & 2.37597000 & 1.17160300 \\ \mathrm{~N} & -2.54992900 & -0.07521800 & -0.03612000 \\ \mathrm{H} & -2.94456900 & -0.05726700 & -0.97874600 \\ \mathrm{H} & -2.95045800 & -0.91092700 & 0.39474300 \\ \mathrm{O} & 2.77792800 & 0.02557200 & -0.00223700 \\ \mathrm{H} & 3.40025600 & -0.13867900 & -0.73006200 \\ \mathrm{H} & 3.36410500 & 0.21990700 & 0.74770200 \\ \mathrm{H} & 0.05320000 & 1.45123500 & 2.28524100 \\ \mathrm{H} & -0.30810500 & 0.98552200 & -2.49832900 \\ \mathrm{H} & -3.00378000 & 0.70188400 & 0.44826000 \\ \mathrm{H} & 0.21423200 & -1.41298900 & -2.26845000 \\ \mathrm{~N} & 0.07411300 & -1.31656600 & 1.61035700 \\ \mathrm{H} & -0.46868800 & -2.17863500 & 1.53432800 \\ \mathrm{H} & 1.05813900 & -1.59238200 & 1.64720400 \\ \mathrm{H} & -0.15187800 & -0.93621000 & 2.53063800 \\ & & & \end{array}$

$\begin{array}{lrrr}\mathrm{Cu}\left(\mathrm{H}_{2} \mathrm{O}\right)_{5}{ }^{2+}(\mathbf{1 2}), \mathrm{E}_{\mathrm{e}}=-2021.9568635, \mathrm{ZPE}=0.1241172 & \\ \mathrm{Cu} & 0.00608900 & 0.01444200 & -0.17582900 \\ \mathrm{O} & 0.76230200 & 1.84265100 & -0.36502600 \\ \mathrm{H} & 0.28769700 & 2.69141100 & -0.33489100 \\ \mathrm{O} & -1.88593800 & 0.71660400 & -0.34915000 \\ \mathrm{H} & -2.27327600 & 1.15384500 & -1.12937400 \\ \mathrm{O} & -0.75119200 & -1.79824500 & -0.49564900 \\ \mathrm{H} & -0.25489500 & -2.59261900 & -0.76018400 \\ \mathrm{O} & -0.04937700 & -0.13424000 & 2.00836200 \\ \mathrm{H} & 0.26076000 & 0.51195100 & 2.66570900 \\ \mathrm{O} & 1.90424600 & -0.67044900 & -0.34842200 \\ \mathrm{H} & 2.35922600 & -0.93209400 & -1.16961600 \\ \mathrm{H} & -2.41202600 & 0.96704500 & 0.43226900 \\ \mathrm{H} & 1.71946400 & 2.02030400 & -0.38477900 \\ \mathrm{H} & -1.68580600 & -1.92386100 & -0.73991100 \\ \mathrm{H} & 2.37469200 & -1.07578800 & 0.40278100 \\ \mathrm{H} & -0.39274300 & -0.88959600 & 2.51612200\end{array}$

$\begin{array}{crrr}\mathrm{Cu}\left(\mathrm{H}_{2} \mathrm{O}\right)_{3}\left(\mathrm{NH}_{3}\right)_{2}{ }^{2+}(\mathbf{1 3}), \mathrm{E}_{\mathrm{e}}= & -1982.2552997, \mathrm{ZPE}=0.1522044 & \\ \mathrm{Cu} & 0.00000000 & 0.00000000 & 0.10986400 \\ \mathrm{O} & 0.00000000 & 2.06090200 & 0.74061200 \\ \mathrm{H} & 0.18761700 & 2.38729700 & 1.63815200 \\ \mathrm{O} & 0.00000000 & -2.06090200 & 0.74061200 \\ \mathrm{H} & 0.34355800 & -2.82074900 & 0.23927300\end{array}$




$\begin{array}{lrrr}\mathrm{O} & 0.00000000 & 0.00000000 & -2.10640100 \\ \mathrm{H} & 0.39457500 & 0.66601900 & -2.69522300 \\ \mathrm{H} & -0.34355800 & 2.82074900 & 0.23927300 \\ \mathrm{H} & -0.39457500 & -0.66601900 & -2.69522300 \\ \mathrm{H} & -0.18761700 & -2.38729700 & 1.63815200 \\ \mathrm{~N} & 2.00258800 & 0.02431800 & 0.17043900 \\ \mathrm{H} & 2.40701300 & -0.53101600 & -0.58751100 \\ \mathrm{H} & 2.37330400 & 0.97303600 & 0.07611200 \\ \mathrm{~N} & -2.00258800 & -0.02431800 & 0.17043900 \\ \mathrm{H} & -2.40701300 & 0.53101600 & -0.58751100 \\ \mathrm{H} & -2.37330400 & -0.97303600 & 0.07611200 \\ \mathrm{H} & 2.37671300 & -0.35545700 & 1.04379900 \\ \mathrm{H} & -2.37671300 & 0.35545700 & 1.04379900\end{array}$

$\begin{array}{lrrr}\mathrm{Cu}\left(\mathrm{H}_{2} \mathrm{O}\right)_{2}\left(\mathrm{NH}_{3}\right)_{3}{ }^{2+}(\mathbf{1 4}), \mathrm{E}_{\mathrm{e}}= & -1962.3996609, \mathrm{ZPE}=0.1664368 & \\ \mathrm{Cu} & -0.10413800 & -0.00026900 & -0.16158000 \\ \mathrm{O} & 0.41891700 & 0.00270800 & 2.09245600 \\ \mathrm{H} & -0.21466700 & 0.02827700 & 2.82945600 \\ \mathrm{H} & 1.29122500 & -0.03497200 & 2.51960000 \\ \mathrm{~N} & 0.01995900 & -2.02398400 & -0.23177100 \\ \mathrm{H} & -0.29363900 & -2.45525300 & 0.64097400 \\ \mathrm{H} & -0.49215100 & -2.47874000 & -0.99122000 \\ \mathrm{~N} & -0.01290900 & 2.02536400 & -0.23190100 \\ \mathrm{H} & -0.27110700 & 2.44504100 & 0.66439600 \\ \mathrm{H} & 0.95369400 & 2.30496900 & -0.41393200 \\ \mathrm{H} & 1.00059100 & -2.28925000 & -0.34887300 \\ \mathrm{H} & -0.58170100 & 2.47963200 & -0.95002500 \\ \mathrm{~N} & -2.17561300 & -0.01718600 & -0.36797300 \\ \mathrm{H} & -2.45368400 & -0.00284800 & -1.35320200 \\ \mathrm{H} & -2.61625200 & -0.84433800 & 0.04098100 \\ \mathrm{H} & -2.63082800 & 0.78734300 & 0.06927400 \\ \mathrm{O} & 2.00614100 & 0.01368800 & -0.85260600 \\ \mathrm{H} & 2.28079500 & -0.00697900 & -1.78608300 \\ \mathrm{H} & 2.82719800 & 0.05439200 & -0.33280500\end{array}$

$\begin{array}{lrrr}\mathrm{Cu}\left(\mathrm{H}_{2} \mathrm{O}\right)\left(\mathrm{NH}_{3}\right)_{4}{ }^{2+}(\mathbf{1 5}), \mathrm{E}_{\mathrm{e}}= & -1942.540063, \mathrm{ZPE}=0.1810138 & \\ \mathrm{Cu} & -0.02162100 & 0.00781400 & -0.22743500 \\ \mathrm{~N} & -2.05993000 & -0.42658000 & -0.45786300 \\ \mathrm{H} & -2.24261800 & -0.87476300 & -1.35977400 \\ \mathrm{H} & -2.41381000 & -1.06788200 & 0.25563600 \\ \mathrm{~N} & 1.98537500 & 0.44629000 & -0.65462700 \\ \mathrm{H} & 2.37913000 & 1.17657900 & -0.05749200 \\ \mathrm{H} & 2.62397200 & -0.34649300 & -0.56245500 \\ \mathrm{~N} & -0.46737000 & 2.03630500 & -0.18610500 \\ \mathrm{H} & -1.10330800 & 2.23442900 & 0.59014200 \\ \mathrm{H} & 0.33638100 & 2.64999600 & -0.04006000 \\ \mathrm{~N} & 0.42012500 & -2.02019000 & -0.29942600\end{array}$




$\begin{array}{rrrr}\mathrm{H} & 1.00404000 & -2.25198500 & 0.50816500 \\ \mathrm{H} & -0.38462200 & -2.64764800 & -0.24590800 \\ \mathrm{O} & 0.17867400 & -0.06052800 & 2.17144200 \\ \mathrm{H} & -0.45735900 & -0.41877400 & 2.81336400 \\ \mathrm{H} & 0.88111100 & 0.32428500 & 2.72209700 \\ \mathrm{H} & -0.92256000 & 2.37571800 & -1.03719200 \\ \mathrm{H} & 2.09597500 & 0.77917000 & -1.61657400 \\ \mathrm{H} & -2.68200700 & 0.38369300 & -0.42574900 \\ \mathrm{H} & 0.93588600 & -2.30946700 & -1.13397800\end{array}$

$\mathrm{Cu}\left(\mathrm{NH}_{3}\right)_{4}{ }^{2+}(\mathbf{1 6}), \mathrm{E}_{\mathrm{e}}=-1866.0524907, \mathrm{ZPE}=0.1572848$

$\begin{array}{lrrr}\mathrm{Cu} & -0.00229400 & -0.00168800 & -0.00149100 \\ \mathrm{~N} & -1.45164500 & -1.41926200 & 0.41031600 \\ \mathrm{~N} & 1.42187000 & -1.44552900 & -0.41019400 \\ \mathrm{H} & 1.03489700 & -2.25786400 & -0.89810900 \\ \mathrm{H} & 2.16814300 & -1.09664700 & -1.01811300 \\ \mathrm{~N} & -1.41440200 & 1.45346700 & -0.40974500 \\ \mathrm{H} & -1.79917200 & 1.90080400 & 0.42726200 \\ \mathrm{H} & -1.03857600 & 2.21090700 & -0.98700100 \\ \mathrm{~N} & 1.44935400 & 1.41513900 & 0.41353800 \\ \mathrm{H} & 1.08357700 & 2.22537800 & 0.92091600 \\ \mathrm{H} & 2.19896200 & 1.04369000 & 1.00374400 \\ \mathrm{H} & 1.88616000 & -1.81389100 & 0.42496200 \\ \mathrm{H} & -2.21860500 & 1.09232900 & -0.93008400 \\ \mathrm{H} & 1.90850000 & 1.79040200 & -0.42133000 \\ \mathrm{H} & -1.08793000 & -2.21368800 & 0.94368200 \\ \mathrm{H} & -2.21708600 & -1.04227400 & 0.97604800 \\ \mathrm{H} & -1.88860400 & -1.81688800 & -0.42615100\end{array}$

$\mathrm{Cu}\left(\mathrm{H}_{2} \mathrm{O}\right)_{4}\left(\mathrm{NH}_{2} \mathrm{CH}_{3}\right)_{2}{ }^{2+}(\mathbf{1 7}), \mathrm{E}_{\mathrm{e}}=-2137.376479, \mathrm{ZPE}=0.2338408$

$\begin{array}{lrrr}\mathrm{Cu} & 0.00029900 & 0.05016500 & 0.04199900 \\ \mathrm{O} & -0.00091200 & 1.81955600 & 1.52934800 \\ \mathrm{H} & -0.56514000 & 2.61009800 & 1.49871800 \\ \mathrm{O} & 0.00565500 & -1.66634700 & -1.40622800 \\ \mathrm{H} & -0.15350000 & -2.60327400 & -1.20578000 \\ \mathrm{O} & 0.80479800 & -1.34270200 & 1.69399800 \\ \mathrm{H} & 1.47732900 & -2.03090800 & 1.56211000 \\ \mathrm{O} & -0.81554100 & 1.46067000 & -1.58750400 \\ \mathrm{H} & -1.48617500 & 1.22112100 & -2.24795800 \\ \mathrm{~N} & 1.80952100 & 0.64768600 & -0.60420300 \\ \mathrm{H} & 1.69991100 & 0.82851900 & -1.60378500 \\ \mathrm{H} & 1.97521200 & 1.56160700 & -0.17763900 \\ \mathrm{~N} & -1.80805900 & -0.49215100 & 0.73641400 \\ \mathrm{H} & -1.69389100 & -1.44772500 & 1.07956500 \\ \mathrm{H} & -1.97752300 & 0.07840700 & 1.56733600 \\ \mathrm{H} & 0.70411500 & -1.26313000 & 2.65701900 \\ \mathrm{H} & 0.15937800 & -1.62554000 & -2.36438900\end{array}$




$\begin{array}{lrrr}\mathrm{H} & -0.69214100 & 2.41961100 & -1.68226200 \\ \mathrm{H} & 0.55566100 & 1.92911500 & 2.31834100 \\ \mathrm{C} & -3.01377200 & -0.43180900 & -0.14903200 \\ \mathrm{H} & -3.21533700 & 0.60545300 & -0.41577900 \\ \mathrm{H} & -2.83107200 & -1.01957900 & -1.04946400 \\ \mathrm{H} & -3.88665200 & -0.83877500 & 0.36857000 \\ \mathrm{C} & 3.01655500 & -0.21280000 & -0.39374900 \\ \mathrm{H} & 3.21449900 & -0.30364300 & 0.67407000 \\ \mathrm{H} & 2.83762600 & -1.19873200 & -0.82444000 \\ \mathrm{H} & 3.89010300 & 0.23208800 & -0.87791100\end{array}$

$\mathrm{Cu}\left(\mathrm{H}_{2} \mathrm{O}\right)_{2}\left(\mathrm{NH}_{2} \mathrm{CH}_{3}\right)_{4}{ }^{2+}(\mathbf{1 8}), \mathrm{E}_{\mathrm{e}}=-2176.2851173, \mathrm{ZPE}=0.3196518$

$\begin{array}{lrrr}\mathrm{Cu} & -0.06994900 & -0.00127600 & 0.02223700 \\ \mathrm{O} & -1.26960400 & 0.26107400 & -2.39980200 \\ \mathrm{H} & -2.07870300 & -0.16598500 & -2.72440500 \\ \mathrm{O} & 1.29270200 & -0.06886800 & 2.76874800 \\ \mathrm{H} & 1.17287600 & 0.40941900 & 3.60587900 \\ \mathrm{H} & 1.95028800 & -0.74916400 & 2.98728100 \\ \mathrm{H} & -1.03692400 & 0.89211200 & -3.09987700 \\ \mathrm{~N} & -1.11600800 & -1.62327200 & 0.84688700 \\ \mathrm{H} & -1.23543600 & -1.40147600 & 1.83762600 \\ \mathrm{~N} & 1.42908300 & 1.45179700 & 0.09660000 \\ \mathrm{H} & 1.83206800 & 1.22636500 & 1.01245300 \\ \mathrm{H} & -0.48974700 & -2.42901800 & 0.84864200 \\ \mathrm{H} & 0.98525300 & 2.36260400 & 0.20977300 \\ \mathrm{~N} & -1.43634700 & 1.32596000 & 0.87429400 \\ \mathrm{H} & -2.28190900 & 0.79830900 & 1.09279900 \\ \mathrm{H} & -1.07754600 & 1.62859700 & 1.78187800 \\ \mathrm{~N} & 1.02900000 & -1.25804400 & -1.19423700 \\ \mathrm{H} & 1.47537000 & -0.65451000 & -1.88552000 \\ \mathrm{H} & 0.34782900 & -1.79451700 & -1.73357800 \\ \mathrm{C} & -2.43009000 & -2.05619400 & 0.27343600 \\ \mathrm{H} & -3.13046900 & -1.21900400 & 0.26780600 \\ \mathrm{H} & -2.28417400 & -2.39592200 & -0.75287000 \\ \mathrm{H} & -2.86647000 & -2.87213000 & 0.85495200 \\ \mathrm{C} & 2.52192300 & 1.60219500 & -0.91161800 \\ \mathrm{H} & 3.12090400 & 0.69133500 & -0.95235000 \\ \mathrm{H} & 2.09374000 & 1.80395100 & -1.89536900 \\ \mathrm{H} & 3.18610800 & 2.42924600 & -0.64723000 \\ \mathrm{C} & 2.05831600 & -2.18016700 & -0.62428800 \\ \mathrm{H} & 2.78793000 & -1.60755500 & -0.04966900 \\ \mathrm{H} & 1.58503000 & -2.90803800 & 0.03727400 \\ \mathrm{H} & 2.58033200 & -2.72625000 & -1.41434100 \\ \mathrm{C} & -1.85874800 & 2.53471000 & 0.09181900 \\ \mathrm{H} & -0.99720000 & 3.17302200 & -0.11084600 \\ \mathrm{H} & -2.28758700 & 2.21043200 & -0.85541100 \\ \mathrm{H} & -2.59631800 & 3.11917900 & 0.64777800 \\ & & & \\ \mathrm{H} & & & \\ & & & \end{array}$




$\begin{array}{crrr}\mathrm{Cu}\left(\mathrm{H}_{2} \mathrm{O}\right)_{3}\left(\mathrm{NH}_{2} \mathrm{CH}_{3}\right)_{2}{ }^{2+}(\mathbf{1 9}), \mathrm{E}_{\mathrm{e}}=-2060.8917485, \mathrm{ZPE}=0.2092903 & \\ \mathrm{Cu} & -0.01940700 & -0.01926500 & -0.03175900 \\ \mathrm{O} & -0.55245300 & -1.87237000 & -1.08445800 \\ \mathrm{H} & -0.69908500 & -1.98316500 & -2.03957200 \\ \mathrm{O} & 0.10008800 & 2.14325500 & -0.11407700 \\ \mathrm{H} & 0.56918800 & 2.71711500 & 0.51510300 \\ \mathrm{O} & 0.54917300 & -0.97634200 & 1.90318900 \\ \mathrm{H} & 1.00226100 & -1.82804800 & 2.02214200 \\ \mathrm{H} & -0.86228000 & -2.69533200 & -0.66948000 \\ \mathrm{H} & 0.49504600 & -0.58726600 & 2.79251900 \\ \mathrm{H} & -0.15384000 & 2.70889000 & -0.86314400 \\ \mathrm{~N} & 1.81455000 & -0.20907200 & -0.83447400 \\ \mathrm{H} & 1.98829000 & -1.21208000 & -0.92998600 \\ \mathrm{~N} & -1.89737400 & 0.19702000 & 0.64020300 \\ \mathrm{H} & -2.15121100 & -0.64171100 & 1.16646100 \\ \mathrm{H} & -1.90299900 & 0.96435100 & 1.31574200 \\ \mathrm{H} & 1.79224000 & 0.15114500 & -1.79194200 \\ \mathrm{C} & 2.97384000 & 0.42307500 & -0.11998100 \\ \mathrm{H} & 2.85856300 & 1.50655700 & -0.13089400 \\ \mathrm{H} & 3.00626400 & 0.06463300 & 0.90894800 \\ \mathrm{H} & 3.91089100 & 0.16424500 & -0.62014900 \\ \mathrm{C} & -2.94983900 & 0.46827400 & -0.39482600 \\ \mathrm{H} & -2.71331700 & 1.39366200 & -0.92183300 \\ \mathrm{H} & -2.98704200 & -0.36059700 & -1.10150400 \\ \mathrm{H} & -3.92887000 & 0.57621300 & 0.08009400\end{array}$

$\begin{array}{ccrr}\mathrm{Cu}\left(\mathrm{H}_{2} \mathrm{O}\right)_{5}\left[\mathrm{~N}\left(\mathrm{CH}_{3}\right)_{3}\right]^{2+}(\mathbf{2 0}), \mathrm{E}_{\mathrm{e}}=-2196.5518447, \mathrm{ZPE}=0.2465351 & \\ \mathrm{Cu} & -0.41343200 & 0.00072800 & 0.02649000 \\ \mathrm{~N} & 1.62709600 & 0.00344700 & -0.03986100 \\ \mathrm{O} & -0.85747500 & 1.76754000 & -1.29914100 \\ \mathrm{H} & -0.33383000 & 2.49560300 & -1.67029100 \\ \mathrm{O} & -0.70519100 & 1.37968200 & 1.76399300 \\ \mathrm{H} & -0.67526500 & 1.15278800 & 2.70786000 \\ \mathrm{O} & -0.89779200 & -1.22382600 & -1.81326900 \\ \mathrm{H} & -0.86401800 & -2.19276300 & -1.87214400 \\ \mathrm{O} & -0.59822900 & -1.82743200 & 1.26796600 \\ \mathrm{H} & -1.51721800 & -2.02649500 & 1.51107200 \\ \mathrm{H} & -0.91284200 & -0.89437900 & -2.72667200 \\ \mathrm{H} & -1.78037500 & 1.95656500 & -1.53279000 \\ \mathrm{H} & -0.81604400 & 2.34302100 & 1.71523300 \\ \mathrm{H} & -0.04622000 & -2.48845800 & 1.71535800 \\ \mathrm{C} & 2.09849300 & 1.20562600 & -0.78771500 \\ \mathrm{H} & 1.75621700 & 2.11121100 & -0.28418100 \\ \mathrm{H} & 3.19381900 & 1.21345100 & -0.80733900 \\ \mathrm{H} & 1.73441200 & 1.16881500 & -1.81501700 \\ \mathrm{C} & 2.15781700 & 0.04076700 & 1.35748300\end{array}$




$\begin{array}{rrrr}\mathrm{H} & 3.25135500 & -0.01247900 & 1.32687800 \\ \mathrm{H} & 1.85968300 & 0.97001200 & 1.83957700 \\ \mathrm{H} & 1.78281000 & -0.80991500 & 1.92599800 \\ \mathrm{C} & 2.08899400 & -1.23813200 & -0.73134400 \\ \mathrm{H} & 1.74506800 & -1.23676300 & -1.76432900 \\ \mathrm{H} & 3.18386800 & -1.26947300 & -0.71785100 \\ \mathrm{H} & 1.70525300 & -2.11879300 & -0.21535300 \\ \mathrm{O} & -2.51586000 & -0.13917800 & 0.19163100 \\ \mathrm{H} & -3.07070700 & 0.42577300 & 0.75345000 \\ \mathrm{H} & -3.07155900 & -0.53682800 & -0.49863900\end{array}$

\section{$\mathrm{Cu}\left(\mathrm{H}_{2} \mathrm{O}\right)_{3}\left(\mathrm{NH}_{2} \mathrm{CH}_{3}\right)\left[\mathrm{N}\left(\mathrm{CH}_{3}\right)_{3}\right]^{2+}(\mathbf{2 1}), \mathrm{E}_{\mathrm{e}}=-2139.5263895, \mathrm{ZPE}=0.2644783$}

$\begin{array}{lrrr}\mathrm{Cu} & 0.30212800 & 0.11346300 & -0.05500800 \\ \mathrm{~N} & 2.30278400 & 0.47550400 & -0.19845100 \\ \mathrm{~N} & -1.70805600 & -0.19534900 & 0.08741300 \\ \mathrm{O} & 0.56090200 & -1.63028600 & -1.39634600 \\ \mathrm{H} & 0.74682600 & -2.54503100 & -1.12647700 \\ \mathrm{O} & 0.06040100 & 2.13693000 & -1.02274700 \\ \mathrm{H} & 0.09507900 & 3.00258400 & -0.58223500 \\ \mathrm{O} & 0.56013600 & 0.10134000 & 2.15530800 \\ \mathrm{H} & 0.84531700 & -0.65230400 & 2.69812800 \\ \mathrm{H} & -0.04583000 & 2.33083000 & -1.96916800 \\ \mathrm{H} & 0.71012500 & -1.60164100 & -2.35630000 \\ \mathrm{H} & 0.53551800 & 0.86544300 & 2.75521100 \\ \mathrm{C} & -2.31487400 & -0.17087500 & -1.27830300 \\ \mathrm{H} & -1.87061000 & -0.95038400 & -1.89662100 \\ \mathrm{H} & -3.39167300 & -0.35689600 & -1.20093800 \\ \mathrm{H} & -2.16633100 & 0.80924800 & -1.73177900 \\ \mathrm{C} & -1.94599900 & -1.52772600 & 0.72174100 \\ \mathrm{H} & -3.02333600 & -1.69885700 & 0.82287900 \\ \mathrm{H} & -1.53122400 & -2.31726400 & 0.09477400 \\ \mathrm{H} & -1.49866300 & -1.55212600 & 1.71598900 \\ \mathrm{C} & -2.32168700 & 0.87904100 & 0.92554900 \\ \mathrm{H} & -2.13544700 & 1.85321900 & 0.47272900 \\ \mathrm{H} & -3.40436200 & 0.72262600 & 0.98255700 \\ \mathrm{H} & -1.91287600 & 0.84177300 & 1.93471000 \\ \mathrm{H} & 2.50779900 & 0.72472800 & -1.16796000 \\ \mathrm{H} & 2.49229500 & 1.32608200 & 0.33501300 \\ \mathrm{C} & 3.27170600 & -0.57965200 & 0.23998100 \\ \mathrm{H} & 3.09742900 & -0.82084700 & 1.28895300 \\ \mathrm{H} & 3.13792600 & -1.47322700 & -0.36894100 \\ \mathrm{H} & 40084300 & -0.22806200 & 0.12844500\end{array}$

$\mathrm{Cu}\left(\mathrm{NH}_{2} \mathrm{CH}_{3}\right)_{3}\left[\mathrm{~N}_{(}\left(\mathrm{CH}_{3}\right)_{3}\right]^{2+}(\mathbf{2 2}), \mathrm{E}_{\mathrm{e}}=-2101.9552556, \mathrm{ZPE}=0.3268502$

$\begin{array}{lrrr}\mathrm{Cu} & -0.27495400 & -0.02215000 & -0.12089900 \\ \mathrm{~N} & 1.75802000 & -0.24664600 & 0.33411800 \\ \mathrm{~N} & -0.13019100 & 1.65335700 & -1.36954800\end{array}$




$\begin{array}{lrrr}\mathrm{H} & -0.79159300 & 1.48682900 & -2.13252200 \\ \mathrm{~N} & -0.72619400 & -2.04720100 & -0.32097500 \\ \mathrm{H} & -1.19315600 & -2.40754500 & 0.51339900 \\ \mathrm{~N} & -2.15883200 & 0.45282700 & 0.63237000 \\ \mathrm{H} & -2.43305400 & 1.39263600 & 0.34417300 \\ \mathrm{H} & 0.78417700 & 1.63886100 & -1.82403000 \\ \mathrm{H} & -2.88217600 & -0.15653900 & 0.24728900 \\ \mathrm{H} & 0.12450200 & -2.60442800 & -0.40198500 \\ \mathrm{C} & -0.36640000 & 3.04654200 & -0.85651300 \\ \mathrm{H} & -0.20172500 & 3.78276100 & -1.64698200 \\ \mathrm{H} & -1.39606000 & 3.14557100 & -0.51004300 \\ \mathrm{H} & 0.31074000 & 3.25550400 & -0.02856300 \\ \mathrm{C} & -2.23352600 & 0.38811000 & 2.13350400 \\ \mathrm{H} & -1.53357500 & 1.10636500 & 2.56346800 \\ \mathrm{H} & -3.24107300 & 0.62593600 & 2.48443900 \\ \mathrm{H} & -1.97316300 & -0.61605400 & 2.47337700 \\ \mathrm{C} & -1.58142900 & -2.34613500 & -1.52059400 \\ \mathrm{H} & -1.81252700 & -3.41294800 & -1.57818300 \\ \mathrm{H} & -2.51923500 & -1.79103300 & -1.46031200 \\ \mathrm{H} & -1.05004100 & -2.05130300 & -2.42688400 \\ \mathrm{C} & 1.85279800 & -1.16450400 & 1.51203800 \\ \mathrm{H} & 1.52090600 & -2.16920100 & 1.24846200 \\ \mathrm{H} & 2.89297200 & -1.23143900 & 1.84941900 \\ \mathrm{H} & 1.24760200 & -0.78035400 & 2.33629500 \\ \mathrm{C} & 2.30712600 & 1.08373700 & 0.73832100 \\ \mathrm{H} & 2.33058700 & 1.76351800 & -0.11352300 \\ \mathrm{H} & 1.70217000 & 1.51259400 & 1.54048500 \\ \mathrm{H} & 3.33305800 & 0.96711200 & 1.10487200 \\ \mathrm{C} & 2.56172100 & -0.79254700 & -0.79930200 \\ \mathrm{H} & 3.61180000 & -0.89254500 & -0.50330300 \\ \mathrm{H} & 2.19266600 & -1.77752200 & -1.09061400 \\ \mathrm{H} & 2.50850800 & -0.12201500 & -1.65914500\end{array}$

$\begin{array}{crrr}\mathrm{Cu}\left(\mathrm{H}_{2} \mathrm{O}\right)_{2}\left[\mathrm{~N}\left(\mathrm{CH}_{3}\right)_{3}\right]_{2}{ }^{2+}(\mathbf{2 3}), \mathrm{E}_{\mathrm{e}}=-2141.6762387, \mathrm{ZPE}=0.2945481 & \\ \mathrm{Cu} & -0.00000700 & -0.00005300 & 0.10046000 \\ \mathrm{~N} & -1.96463400 & 0.00386300 & -0.33503300 \\ \mathrm{~N} & 1.96478700 & -0.00385400 & -0.33476500 \\ \mathrm{C} & -2.18588200 & 1.20146700 & -1.20431100 \\ \mathrm{H} & -3.22764500 & 1.22910400 & -1.54440400 \\ \mathrm{H} & -1.98241600 & 2.11248400 & -0.63972500 \\ \mathrm{H} & -1.54287400 & 1.15148900 & -2.08744900 \\ \mathrm{C} & -2.84950100 & 0.06640800 & 0.86639200 \\ \mathrm{H} & -3.89868800 & 0.03010300 & 0.55400600 \\ \mathrm{H} & -2.64422800 & -0.78266900 & 1.51928800 \\ \mathrm{H} & -2.67687800 & 1.00066400 & 1.40148700 \\ \mathrm{C} & -2.23935500 & -1.24422000 & -1.11194600 \\ \mathrm{H} & -3.26928800 & -1.23138700 & -1.48673100\end{array}$




$\begin{array}{lrrr}\mathrm{H} & -1.56682900 & -1.31320500 & -1.97142200 \\ \mathrm{H} & -2.12154500 & -2.11739900 & -0.46883400 \\ \mathrm{C} & 2.84953800 & -0.06990300 & 0.86657800 \\ \mathrm{H} & 2.67614100 & -1.00521700 & 1.39956400 \\ \mathrm{H} & 3.89878100 & -0.03365700 & 0.55437200 \\ \mathrm{H} & 2.64489600 & 0.77782600 & 1.52145400 \\ \mathrm{C} & 2.24102700 & 1.24557500 & -1.10897500 \\ \mathrm{H} & 3.27083600 & 1.23214300 & -1.48405600 \\ \mathrm{H} & 1.56833600 & 1.31741600 & -1.96808300 \\ \mathrm{H} & 2.12461400 & 2.11748000 & -0.46389500 \\ \mathrm{C} & 2.18500600 & -1.19975900 & -1.20668300 \\ \mathrm{H} & 3.22682200 & -1.22781900 & -1.54657400 \\ \mathrm{H} & 1.98044400 & -2.11186500 & -0.64422800 \\ \mathrm{H} & 1.54228200 & -1.14708000 & -2.08986900 \\ \mathrm{O} & 0.01548900 & 1.75248100 & 1.36084400 \\ \mathrm{H} & -0.21292200 & 1.76094500 & 2.30549400 \\ \mathrm{O} & -0.01663600 & -1.75194500 & 1.36130200 \\ \mathrm{H} & -0.16982600 & -2.67868700 & 1.11269800 \\ \mathrm{H} & 0.21058300 & -1.75947200 & 2.30625100 \\ \mathrm{H} & 0.17270600 & 2.67858600 & 1.11239900\end{array}$

$\begin{array}{crrr}\mathrm{Cu}\left(\mathrm{H}_{2} \mathrm{O}\right)_{3}\left[\left(\mathrm{CH}_{3}\right)_{2} \mathrm{NCH}_{2} \mathrm{CH}_{2} \mathrm{~N}\left(\mathrm{CH}_{3}\right)_{2}\right]^{2+}(\mathbf{2 4}), \mathrm{E}_{\mathrm{e}}=-2216.9625664, \mathrm{ZPE}=0.3020619 \\ \mathrm{C} & 0.16604600 & 2.20619600 & 0.50970900 \\ \mathrm{C} & 1.47272100 & 1.78939400 & -0.14369900 \\ \mathrm{H} & 0.20866100 & 2.07189100 & 1.59197600 \\ \mathrm{H} & -0.03757100 & 3.26598800 & 0.32053500 \\ \mathrm{H} & 2.31430100 & 2.33341600 & 0.29897500 \\ \mathrm{H} & 1.46753800 & 2.01457900 & -1.21173100 \\ \mathrm{Cu} & -0.20471700 & -0.53070000 & -0.17182900 \\ \mathrm{O} & 0.47248600 & -2.42732700 & -0.76315500 \\ \mathrm{H} & -0.23295900 & -2.98316000 & -1.13559400 \\ \mathrm{O} & -0.79084100 & -1.67126100 & 1.78612900 \\ \mathrm{H} & -0.36648200 & -2.53465800 & 1.92180700 \\ \mathrm{O} & -2.08369500 & -1.45909600 & -0.75557700 \\ \mathrm{H} & -2.65562400 & -1.40273800 & -1.53876900 \\ \mathrm{~N} & 1.68271500 & 0.31087800 & 0.01369600 \\ \mathrm{~N} & -0.96171200 & 1.36578700 & -0.01248900 \\ \mathrm{H} & -2.60344400 & -1.86644000 & -0.04205700 \\ \mathrm{H} & 1.30086100 & -2.69861200 & -1.19017900 \\ \mathrm{H} & -1.14670500 & -1.42044600 & 2.65404100 \\ \mathrm{C} & -1.37919500 & 1.80700700 & -1.38070500 \\ \mathrm{H} & -0.55252400 & 1.72292700 & -2.08723000 \\ \mathrm{H} & -2.20937000 & 1.18975100 & -1.72444100 \\ \mathrm{H} & -1.71212800 & 2.85018200 & -1.34885700 \\ \mathrm{C} & -2.13038300 & 1.44646000 & 0.91137400 \\ \mathrm{H} & -2.48263800 & 2.48207600 & 0.97674200 \\ \mathrm{H} & -2.94194000 & 0.82438100 & 0.53431200 \\ & & & \end{array}$




$\begin{array}{rrrr}\mathrm{H} & -1.84066500 & 1.11364200 & 1.90922800 \\ \mathrm{C} & 2.19632700 & -0.02683600 & 1.37738000 \\ \mathrm{H} & 1.52317200 & 0.35055000 & 2.14690100 \\ \mathrm{H} & 2.27756900 & -1.10992900 & 1.47751300 \\ \mathrm{H} & 3.18745100 & 0.41787400 & 1.51983100 \\ \mathrm{C} & 2.65470800 & -0.16617800 & -1.01451700 \\ \mathrm{H} & 3.56968300 & 0.43484000 & -0.96679900 \\ \mathrm{H} & 2.93141700 & -1.20211400 & -0.81363300 \\ \mathrm{H} & 2.22622100 & -0.07513600 & -2.01441300\end{array}$

$\begin{array}{ccrr}\mathrm{Cu}\left(\mathrm{H}_{2} \mathrm{O}\right)_{3}\left(\mathrm{H}_{2} \mathrm{NCH}_{2} \mathrm{CH}_{2} \mathrm{NHCOCH}_{3}\right)^{2+}(\mathbf{2 5 a}), \mathrm{E}_{\mathrm{e}}=-2212.3078324, \mathrm{ZPE}=0.2274728 \\ \mathrm{Cu} & 0.78100500 & -0.22220900 & -0.22800100 \\ \mathrm{~N} & -1.15307200 & 0.25933600 & -0.71031500 \\ \mathrm{~N} & 1.10758400 & 1.78302200 & -0.17527300 \\ \mathrm{C} & -1.34582700 & 1.70064400 & -0.28785800 \\ \mathrm{C} & -0.10611800 & 2.48117300 & -0.72012400 \\ \mathrm{H} & 1.94495800 & 2.07108800 & -0.68697000 \\ \mathrm{H} & 1.27403200 & 2.05196200 & 0.79839600 \\ \mathrm{H} & -0.02061200 & 2.50460900 & -1.80896800 \\ \mathrm{H} & -0.15715800 & 3.51321600 & -0.36245300 \\ \mathrm{H} & -1.45616600 & 1.71961700 & 0.80014300 \\ \mathrm{H} & -2.25374600 & 2.11395700 & -0.73367800 \\ \mathrm{C} & -1.72902000 & -0.76002200 & 0.20192100 \\ \mathrm{O} & -0.87286200 & -1.27300700 & 0.91437800 \\ \mathrm{C} & -3.18036700 & -1.07061000 & 0.17455300 \\ \mathrm{H} & -3.45982200 & -1.45027000 & -0.81721900 \\ \mathrm{H} & -3.41706300 & -1.81621600 & 0.93378600 \\ \mathrm{H} & -3.76987800 & -0.16096100 & 0.34177000 \\ \mathrm{H} & -1.46903300 & 0.12019900 & -1.67469700 \\ \mathrm{O} & 2.39214600 & -0.71936800 & 0.91112600 \\ \mathrm{H} & 3.25366500 & -1.01113900 & 0.56632700 \\ \mathrm{O} & 0.97465200 & -1.88317300 & -1.54406700 \\ \mathrm{H} & 1.31134100 & -1.89031600 & -2.45605300 \\ \mathrm{H} & 0.98043100 & -2.80688700 & -1.23948000 \\ \mathrm{H} & 2.27715700 & -1.13098400 & 1.78611300 \\ \mathrm{O} & 0.11320400 & 0.49131200 & 1.80365500 \\ \mathrm{H} & -0.82304700 & 0.56424300 & 2.00294300 \\ \mathrm{H} & 0.76509300 & 0.72221200 & 2.46948200\end{array}$

$\begin{array}{crrr}\mathrm{Cu}\left(\mathrm{H}_{2} \mathrm{O}\right)_{3}\left(\mathrm{H}_{2} \mathrm{NCH}_{2} \mathrm{CH}_{2} \mathrm{NHCOCH}_{3}\right)^{2+}(\mathbf{2 5 b}), \mathrm{E}_{\mathrm{e}}=-2212.4002946, \mathrm{ZPE}=0.2277348 \\ \mathrm{Cu} & -0.91537700 & -0.11013300 & -0.10586600 \\ \mathrm{~N} & -0.42189700 & 1.78449800 & 0.31265400 \\ \mathrm{H} & -0.85372300 & 2.34829900 & -0.42376200 \\ \mathrm{C} & 1.00967600 & 2.19984900 & 0.47819000 \\ \mathrm{H} & 1.35708200 & 1.85748100 & 1.45408500 \\ \mathrm{H} & 1.04744200 & 3.29301000 & 0.47688000 \\ \mathrm{H} & -0.92988200 & 2.00402800 & 1.17393300\end{array}$




$\begin{array}{lrrr}\mathrm{C} & 1.91716800 & 1.66002800 & -0.64246100 \\ \mathrm{O} & 0.83357200 & -0.96083700 & 0.03132300 \\ \mathrm{H} & 1.34817300 & 1.51073100 & -1.56735800 \\ \mathrm{H} & 2.68415800 & 2.40260200 & -0.86463100 \\ \mathrm{~N} & 2.63432400 & 0.42450500 & -0.29769100 \\ \mathrm{H} & 3.64752000 & 0.46518100 & -0.31071300 \\ \mathrm{C} & 2.09479700 & -0.75931900 & -0.01360300 \\ \mathrm{C} & 3.00552000 & -1.92545500 & 0.25966900 \\ \mathrm{H} & 2.87244500 & -2.66980900 & -0.53160300 \\ \mathrm{H} & 4.05675200 & -1.63530400 & 0.29768100 \\ \mathrm{H} & 2.72242900 & -2.39207900 & 1.20634900 \\ \mathrm{O} & -1.49084300 & -1.91830700 & -0.87678500 \\ \mathrm{O} & -2.69651300 & 0.62240400 & -1.02237200 \\ \mathrm{H} & -3.49707900 & 1.01254100 & -0.63367000 \\ \mathrm{O} & -1.82547900 & -0.43200000 & 1.95893700 \\ \mathrm{H} & -2.74531900 & -0.44386000 & 2.27069500 \\ \mathrm{H} & -2.94313900 & 0.30002400 & -1.90652800 \\ \mathrm{H} & -0.79320900 & -2.59631000 & -0.88324600 \\ \mathrm{H} & -1.31822700 & -0.90398300 & 2.64006400 \\ \mathrm{H} & -2.32533700 & -2.36241900 & -0.65239700\end{array}$

$\begin{array}{crrr}\mathrm{Cu}\left(\mathrm{H}_{2} \mathrm{O}\right)_{2}\left[\left(\mathrm{CH}_{3}\right)_{2} \mathrm{NCH}_{2} \mathrm{CH}_{2} \mathrm{~N}\left(\mathrm{CH}_{3}\right)_{2}\right]_{2}{ }^{2+}(\mathbf{2 6}), \mathrm{E}_{\mathrm{e}}=-2488.381868, \mathrm{ZPE}=0.5049169 \\ \mathrm{Cu} & -0.00117400 & 0.00033700 & -0.00211700 \\ \mathrm{~N} & 1.77365500 & 1.34010700 & 0.14264400 \\ \mathrm{~N} & -1.52170200 & 1.51539500 & 0.31236900 \\ \mathrm{~N} & -1.77531500 & -1.33893800 & -0.14087500 \\ \mathrm{~N} & 1.51888100 & -1.51457100 & -0.31560500 \\ \mathrm{C} & -2.69176100 & 0.94612100 & -0.42571500 \\ \mathrm{H} & -3.57445900 & 1.57641400 & -0.26039600 \\ \mathrm{H} & -2.45275200 & 0.97451800 & -1.49172300 \\ \mathrm{C} & -2.97704500 & -0.46285500 & 0.03515300 \\ \mathrm{H} & -3.82273600 & -0.87756300 & -0.52381000 \\ \mathrm{H} & -3.26115100 & -0.47176300 & 1.08725500 \\ \mathrm{C} & 2.97425000 & 0.46318500 & -0.03620000 \\ \mathrm{H} & 3.82139000 & 0.87671100 & 0.52150500 \\ \mathrm{H} & 3.25617500 & 0.47251600 & -1.08889900 \\ \mathrm{C} & 2.68794800 & -0.94557800 & 0.42442400 \\ \mathrm{H} & 3.57060900 & -1.57642800 & 0.26121100 \\ \mathrm{H} & 2.44633400 & -0.97290500 & 1.48982700 \\ \mathrm{C} & 1.33285600 & -2.89983000 & 0.19737800 \\ \mathrm{H} & 0.56388000 & -3.41934600 & -0.37023700 \\ \mathrm{H} & 2.26678900 & -3.46311800 & 0.08564900 \\ \mathrm{H} & 1.06376500 & -2.87350000 & 1.25299600 \\ \mathrm{C} & 1.80761900 & -1.59558500 & -1.78117900 \\ \mathrm{H} & 2.78016000 & -2.07186600 & -1.95312900 \\ \mathrm{H} & 1.04199500 & -2.19932200 & -2.26660200 \\ \mathrm{H} & 1.80156100 & -0.60682400 & -2.23767800 \\ & & & \end{array}$




$\begin{array}{lrrr}\mathrm{C} & 1.87700800 & 2.43457300 & -0.86828300 \\ \mathrm{H} & 1.16285300 & 3.22440100 & -0.65742100 \\ \mathrm{H} & 2.88139500 & 2.87280400 & -0.83863200 \\ \mathrm{H} & 1.68727800 & 2.03244400 & -1.86459000 \\ \mathrm{C} & 1.80927800 & 1.91845700 & 1.51748800 \\ \mathrm{H} & 2.72687700 & 2.50131500 & 1.65871200 \\ \mathrm{H} & 0.96025900 & 2.58292300 & 1.66750100 \\ \mathrm{H} & 1.77391500 & 1.11933700 & 2.25937400 \\ \mathrm{C} & -1.87577100 & -2.42901900 & 0.87523900 \\ \mathrm{H} & -2.87992200 & -2.86803200 & 0.85002700 \\ \mathrm{H} & -1.16152500 & -3.21921700 & 0.66619300 \\ \mathrm{H} & -1.68328200 & -2.02231400 & 1.86919200 \\ \mathrm{C} & -1.81191900 & -1.92363000 & -1.51295400 \\ \mathrm{H} & -0.96270500 & -2.58826500 & -1.66098500 \\ \mathrm{H} & -2.72939000 & -2.50749900 & -1.65061900 \\ \mathrm{H} & -1.77766400 & -1.12823400 & -2.25888600 \\ \mathrm{C} & -1.80879000 & 1.59659000 & 1.77821600 \\ \mathrm{H} & -1.80396400 & 0.60766100 & 2.23443700 \\ \mathrm{H} & -1.04208200 & 2.19926300 & 2.26314200 \\ \mathrm{H} & -2.78067200 & 2.07378100 & 1.95143100 \\ \mathrm{C} & -1.33666700 & 2.90081400 & -0.20042500 \\ \mathrm{H} & -2.27108700 & 3.46337700 & -0.08907300 \\ \mathrm{H} & -0.56837600 & 3.42071300 & 0.36775600 \\ \mathrm{H} & -1.06698400 & 2.87547400 & -1.25593000 \\ \mathrm{H} & -0.17560900 & 0.22716900 & -3.99082900 \\ \mathrm{O} & -0.28553700 & 0.83691300 & -3.24418700 \\ \mathrm{H} & -0.46456800 & 1.68979500 & -3.67071100 \\ \mathrm{H} & 0.16555700 & -0.23344800 & 3.98973300 \\ \mathrm{O} & 0.30542800 & -0.84240700 & 3.24747500 \\ \mathrm{H} & 0.49238400 & -1.69021900 & 3.68071100\end{array}$

$\begin{array}{ccrr}\mathrm{Cu}\left(\mathrm{H}_{2} \mathrm{O}\right)\left[\left(\mathrm{CH}_{3}\right)_{2} \mathrm{NCH}_{2} \mathrm{CH}_{2} \mathrm{~N}_{\left.\left(\mathrm{CH}_{3}\right)_{2}\right]_{2}{ }^{2+}(\mathbf{2 7}), \mathrm{E}_{\mathrm{e}}}\right. & -2411.8743555, \mathrm{ZPE}=0.4822798 \\ \mathrm{Cu} & 0.01161200 & 0.03110700 & -0.12981900 \\ \mathrm{~N} & 1.94387100 & -1.13374700 & -0.10266000 \\ \mathrm{~N} & -1.32579400 & -1.64790700 & -0.22127700 \\ \mathrm{~N} & -1.93047000 & 1.19366700 & -0.06676800 \\ \mathrm{~N} & 1.35794500 & 1.71226100 & -0.12243800 \\ \mathrm{C} & -2.57611900 & -1.15242000 & 0.43599600 \\ \mathrm{H} & -3.37378700 & -1.89500600 & 0.31318600 \\ \mathrm{H} & -2.37811000 & -1.06378700 & 1.50748800 \\ \mathrm{C} & -3.01371100 & 0.16447500 & -0.15871900 \\ \mathrm{H} & -3.91221100 & 0.52634100 & 0.35323200 \\ \mathrm{H} & -3.28079200 & 0.03957900 & -1.20803900 \\ \mathrm{C} & 3.03455700 & -0.10813200 & -0.07394000 \\ \mathrm{H} & 3.93134100 & -0.50770900 & -0.55982700 \\ \mathrm{H} & 3.30160700 & 0.08464900 & 0.96500500 \\ \mathrm{C} & 2.59986200 & 1.16749800 & -0.75551300\end{array}$




\begin{tabular}{|c|c|c|c|}
\hline $\mathrm{H}$ & 3.40191300 & 1.91290500 & -0.6927520 \\
\hline $\mathrm{H}$ & 2.39106900 & 1.00416400 & -1.8161880 \\
\hline C & 0.99964600 & 2.94235800 & -0.8870320 \\
\hline $\mathrm{H}$ & 0.19257100 & 3.47538500 & $-0.391397 c$ \\
\hline $\mathrm{H}$ & 1.86492600 & 3.61340100 & -0.932574 \\
\hline $\mathrm{H}$ & 0.70477500 & 2.68362600 & -1.905079 \\
\hline C & 1.62572700 & 2.10269900 & 1.298436 \\
\hline $\mathrm{H}$ & 2.50714500 & 2.75191100 & 1.354074 \\
\hline $\mathrm{H}$ & 0.77348200 & 2.65068100 & 1.6955860 \\
\hline $\mathrm{H}$ & 1.79324200 & 1.22671400 & 1.9249870 \\
\hline C & 2.17031900 & -2.05086900 & 1.055909 \\
\hline $\mathrm{H}$ & 1.54922300 & -2.93717300 & 0.9747530 \\
\hline $\mathrm{H}$ & 3.21731500 & -2.37493300 & 1.073998 \\
\hline $\mathrm{H}$ & 1.94935400 & -1.53420800 & 1.992162 \\
\hline $\mathrm{C}$ & 2.04458800 & -1.90374300 & -1.3773820 \\
\hline $\mathrm{H}$ & 3.02597900 & -2.38614800 & -1.451581 \\
\hline $\mathrm{H}$ & 1.28419400 & -2.68078600 & -1.40995 \\
\hline $\mathrm{H}$ & 1.91619500 & -1.24281000 & -2.237060 \\
\hline C & -2.19717600 & 2.19841000 & -1.141423 \\
\hline $\mathrm{H}$ & -3.25762200 & 2.47552500 & -1.1338970 \\
\hline $\mathrm{H}$ & -1.62087900 & 3.10345500 & -0.977333 \\
\hline $\mathrm{H}$ & -1.95355600 & 1.77979600 & -2.119971 \\
\hline C & -2.00426500 & 1.86397300 & 1.264734 \\
\hline $\mathrm{H}$ & -1.25465500 & 2.65051300 & 1.329468 \\
\hline $\mathrm{H}$ & -2.99014700 & 2.32208400 & 1.405280 \\
\hline $\mathrm{H}$ & -1.83675400 & 1.14495000 & 2.069243 \\
\hline C & -1.57123400 & -1.93240000 & -1.6720800 \\
\hline $\mathrm{H}$ & -1.73563100 & -1.01261700 & -2.233514 \\
\hline $\mathrm{H}$ & -0.70852800 & -2.44334700 & -2.096000 \\
\hline $\mathrm{H}$ & -2.44797000 & -2.57958500 & -1.790888 \\
\hline $\mathrm{C}$ & -0.98128600 & -2.93234900 & 0.456148 \\
\hline $\mathrm{H}$ & -1.84328600 & -3.60860300 & 0.422643 \\
\hline $\mathrm{H}$ & -0.15652200 & -3.42608300 & -0.052166 \\
\hline $\mathrm{H}$ & -0.72426000 & -2.75004900 & 1.500330 \\
\hline $\mathrm{H}$ & -1.07265900 & -0.39842100 & 2.076442 \\
\hline O & -0.11270500 & -0.40304300 & 2.084602 \\
\hline $\mathrm{H}$ & 0.20361600 & -1.30908400 & 2.05947 \\
\hline
\end{tabular}

\section{$\mathrm{Cu}\left(\mathrm{H}_{2} \mathrm{O}\right)_{2}\left(\mathrm{H}_{2} \mathrm{NCH}_{2} \mathrm{CH}_{2} \mathrm{NHCOCH}_{3}\right)_{2}{ }^{2+}(\mathbf{2 8}), \mathrm{E}_{\mathrm{e}}=-2479.3012689, \mathrm{ZPE}=0.3549405$}

$\begin{array}{lrrr}\mathrm{Cu} & 0.00036500 & -0.00017100 & -0.00065200 \\ \mathrm{~N} & -0.65569500 & -1.79499000 & 0.67586500 \\ \mathrm{H} & -0.22453500 & -2.46785100 & 0.04015000 \\ \mathrm{~N} & 0.65609500 & 1.79741100 & -0.67084100 \\ \mathrm{H} & 0.22634000 & 2.46644100 & -0.03010500 \\ \mathrm{H} & -0.17239200 & -1.91144500 & 1.56771500 \\ \mathrm{H} & 0.17096000 & 1.91977100 & -1.56086800 \\ \mathrm{C} & 2.10048200 & 2.12268300 & -0.85008600\end{array}$




\begin{tabular}{|c|c|c|c|}
\hline $\mathrm{H}$ & 2.20644100 & 3.20076200 & -1.0035600 \\
\hline $\mathrm{H}$ & 2.45623200 & 1.62565500 & -1.7539450 \\
\hline $\mathrm{C}$ & 2.94722300 & 1.69871000 & 0.3638360 \\
\hline $\mathrm{H}$ & 3.73483700 & 2.43475300 & 0.5283090 \\
\hline $\mathrm{H}$ & 2.32582600 & 1.67900400 & 1.264764 \\
\hline $\mathrm{N}$ & 3.62700600 & 0.40517700 & 0.204839 \\
\hline $\mathrm{H}$ & 4.63898300 & 0.41056400 & 0.239707 \\
\hline $\mathrm{C}$ & 3.04143700 & -0.78607100 & 0.041812 \\
\hline $\mathrm{C}$ & 3.92900400 & -2.00028500 & -0.0714440 \\
\hline $\mathrm{H}$ & 3.69537400 & -2.68678500 & 0.7471380 \\
\hline $\mathrm{H}$ & 3.71185500 & -2.51918000 & -1.008674 \\
\hline $\mathrm{H}$ & 4.99177900 & -1.75552900 & -0.036708 \\
\hline O & 1.78434200 & -0.94343100 & -0.017083 \\
\hline $\mathrm{C}$ & -2.10042600 & -2.11891500 & 0.8544820 \\
\hline $\mathrm{H}$ & -2.20693300 & -3.19623800 & 1.0127740 \\
\hline $\mathrm{H}$ & -2.45720000 & -1.61769500 & 1.755619 \\
\hline $\mathrm{C}$ & -2.94575700 & -1.70050800 & -0.362343 \\
\hline $\mathrm{H}$ & -3.73357000 & -2.43707800 & -0.523546 \\
\hline $\mathrm{H}$ & -2.32378100 & -1.68584000 & -1.2629490 \\
\hline $\mathrm{N}$ & -3.62551700 & -0.40595100 & -0.2105410 \\
\hline $\mathrm{H}$ & -4.63728300 & -0.41110400 & -0.250767 \\
\hline C & -3.04055700 & 0.78551600 & -0.04651 \\
\hline $\mathrm{C}$ & -3.92857800 & 1.99985300 & 0.061467 \\
\hline $\mathrm{H}$ & -3.71965700 & 2.51629300 & 1.0019660 \\
\hline $\mathrm{H}$ & -3.68733100 & 2.68834600 & -0.753161 \\
\hline $\mathrm{H}$ & -4.99110200 & 1.75562900 & 0.016818 \\
\hline 0 & -1.78374500 & 0.94283900 & 0.0187950 \\
\hline 0 & -0.19740500 & -0.69634100 & -2.539709 \\
\hline $\mathrm{H}$ & 0.50547300 & -1.24596600 & -2.919762 \\
\hline $\mathrm{H}$ & -0.77733700 & -0.48234200 & -3.287387 \\
\hline $\mathrm{H}$ & 0.77456100 & 0.48862200 & 3.295513 \\
\hline 0 & 0.19259300 & 0.69429400 & 2.547056 \\
\hline $\mathrm{H}$ & -0.51459800 & 1.23986400 & 2.924901 \\
\hline
\end{tabular}

$\begin{array}{ccrr}\mathrm{Cu}\left(\mathrm{H}_{2} \mathrm{O}\right)\left(\mathrm{H}_{2} \mathrm{NCH}_{2} \mathrm{CH}_{2} \mathrm{NHCOCH}_{3}\right)_{2}{ }^{2+}(\mathbf{2 9}), \mathrm{E}_{\mathrm{e}}= & -2402.8249994, \mathrm{ZPE}=0.3310918 \\ \mathrm{Cu} & -0.01978000 & -0.03619000 & 0.16119400 \\ \mathrm{~N} & 0.67726900 & -1.86873100 & -0.38949900 \\ \mathrm{H} & 0.50405500 & -2.41372100 & 0.45835700 \\ \mathrm{~N} & -0.73068200 & 1.79161700 & 0.70933000 \\ \mathrm{H} & -0.07033100 & 2.42583700 & 0.25644600 \\ \mathrm{H} & 0.00340200 & -2.21897500 & -1.07254900 \\ \mathrm{H} & -0.52671000 & 1.85392300 & 1.70854000 \\ \mathrm{C} & -2.13320100 & 2.24423800 & 0.46240600 \\ \mathrm{H} & -2.15845400 & 3.33751100 & 0.47209900 \\ \mathrm{H} & -2.76242300 & 1.89514300 & 1.28267600 \\ \mathrm{C} & -2.69107500 & 1.73890200 & -0.88040500 \\ \mathrm{H} & -3.36668300 & 2.48912100 & -1.29310300\end{array}$




$\begin{array}{lrrr}\mathrm{H} & -1.88234100 & 1.60732400 & -1.60830900 \\ \mathrm{~N} & -3.47245100 & 0.49896500 & -0.77730000 \\ \mathrm{H} & -4.45171000 & 0.55031100 & -1.03150600 \\ \mathrm{C} & -3.01415700 & -0.70016300 & -0.40723500 \\ \mathrm{C} & -3.97956400 & -1.85690900 & -0.38885400 \\ \mathrm{H} & -3.64808700 & -2.60703900 & -1.11268500 \\ \mathrm{H} & -3.96350400 & -2.32234600 & 0.59985100 \\ \mathrm{H} & -5.00149000 & -1.55993200 & -0.62978800 \\ \mathrm{O} & -1.80501300 & -0.91241300 & -0.07696000 \\ \mathrm{C} & 2.06212400 & -2.14361800 & -0.87349900 \\ \mathrm{H} & 2.20316800 & -3.22697000 & -0.93750100 \\ \mathrm{H} & 2.16628600 & -1.73786700 & -1.88154500 \\ \mathrm{C} & 3.13820300 & -1.55735300 & 0.05620000 \\ \mathrm{H} & 4.00731100 & -2.21617900 & 0.04973900 \\ \mathrm{H} & 2.77252400 & -1.52244400 & 1.08782600 \\ \mathrm{~N} & 3.63172300 & -0.23033100 & -0.33371500 \\ \mathrm{H} & 4.60935800 & -0.16191900 & -0.59013300 \\ \mathrm{C} & 2.93758100 & 0.91076800 & -0.32268700 \\ \mathrm{C} & 3.65871800 & 2.18409800 & -0.68117200 \\ \mathrm{H} & 3.14397300 & 2.66635900 & -1.51634700 \\ \mathrm{H} & 3.61714800 & 2.86530700 & 0.17362900 \\ \mathrm{H} & 4.70291800 & 2.01900000 & -0.95123900 \\ \mathrm{O} & 1.70691800 & 0.97247100 & -0.01200100 \\ \mathrm{O} & 0.09257800 & -0.71470200 & 2.51782000 \\ \mathrm{H} & -0.64450200 & -1.15500400 & 2.97049000 \\ \mathrm{H} & 0.75103900 & -0.54120400 & 3.20933500\end{array}$

$\begin{array}{crrr}\mathrm{Cu}\left(\mathrm{H}_{2} \mathrm{O}\right)_{2}\left[\mathrm{CH}_{3} \mathrm{NHCOCH}_{2} \mathrm{CH}_{2} \mathrm{~N}\left(\mathrm{CH}_{3}\right) \mathrm{CH}_{2} \mathrm{CH}_{2} \mathrm{~N}\left(\mathrm{CH}_{3}\right) \mathrm{CH}_{2} \mathrm{CH}_{2} \mathrm{CONHCH}_{3}\right]^{2+}(\mathbf{3 0}), \\ \mathrm{E}_{\mathrm{e}}=-2635.3517542, \mathrm{ZPE}= & 0.4468771 & & \\ \mathrm{Cu} & -0.03736000 & -0.22432000 & 0.00717800 \\ \mathrm{~N} & 1.27243200 & -1.77484000 & 0.24605900 \\ \mathrm{~N} & -1.51518100 & -1.64019500 & -0.39547900 \\ \mathrm{C} & 2.52862800 & -1.48054800 & -0.51469700 \\ \mathrm{H} & 3.16496000 & -2.37463000 & -0.49934900 \\ \mathrm{H} & 2.24275100 & -1.27905400 & -1.54842900 \\ \mathrm{C} & 3.35276400 & -0.30680500 & 0.02283200 \\ \mathrm{H} & 4.25673000 & -0.24157000 & -0.58970700 \\ \mathrm{H} & 3.69728200 & -0.50000900 & 1.04435600 \\ \mathrm{C} & 2.64769700 & 1.04192400 & 0.03025600 \\ \mathrm{O} & 1.39020900 & 1.15734800 & 0.14696400 \\ \mathrm{~N} & 3.42678100 & 2.12285000 & -0.04222600 \\ \mathrm{H} & 4.42598000 & 1.97399600 & -0.12260300 \\ \mathrm{C} & -2.82788400 & -1.40781500 & 0.30977600 \\ \mathrm{H} & -3.62741800 & -1.43642500 & -0.43377600 \\ \mathrm{H} & -3.00812700 & -2.23296400 & 1.00142900 \\ \mathrm{C} & -2.90775500 & -0.09536700 & 1.11417400 \\ \mathrm{H} & -3.91726200 & -0.01402400 & 1.52553400\end{array}$




\begin{tabular}{|c|c|c|c|}
\hline $\mathrm{H}$ & -2.20711100 & -0.12100900 & 1.9547970 \\
\hline $\mathrm{C}$ & -2.53887600 & 1.12732100 & 0.3000070 \\
\hline 0 & -1.37742800 & 1.21750100 & $-0.210767 c$ \\
\hline $\mathrm{N}$ & -3.44137100 & 2.09465700 & 0.1458280 \\
\hline $\mathrm{H}$ & -4.34534100 & 1.96229800 & 0.5837780 \\
\hline $\mathrm{C}$ & 0.58505200 & -2.96191600 & -0.3583580 \\
\hline $\mathrm{C}$ & -0.89861000 & -2.94781100 & -0.0023590 \\
\hline $\mathrm{H}$ & 1.04844700 & -3.89057900 & -0.0065710 \\
\hline $\mathrm{H}$ & 0.72393700 & -2.91867800 & -1.4397240 \\
\hline $\mathrm{H}$ & -1.03313200 & -3.07301900 & 1.0734090 \\
\hline $\mathrm{H}$ & -1.41325700 & -3.78017200 & -0.496628 \\
\hline $\mathrm{C}$ & -1.73467800 & -1.58756300 & -1.875708 \\
\hline $\mathrm{H}$ & -0.79145100 & -1.70394100 & -2.406052 \\
\hline $\mathrm{H}$ & -2.42710900 & -2.38048300 & -2.1793600 \\
\hline $\mathrm{H}$ & -2.14860100 & -0.61637400 & -2.1451300 \\
\hline C & 1.55926500 & -2.02747400 & 1.689440 \\
\hline $\mathrm{H}$ & 0.65887000 & -2.38107100 & 2.190311 \\
\hline $\mathrm{H}$ & 2.34140700 & -2.78901400 & 1.788981 \\
\hline $\mathrm{H}$ & 1.86425500 & -1.10659900 & 2.181831 \\
\hline $\mathrm{C}$ & 2.95644800 & 3.51220100 & 0.028231 \\
\hline $\mathrm{H}$ & 1.86896600 & 3.51866800 & -0.0122020 \\
\hline $\mathrm{H}$ & 3.29098900 & 3.97614600 & 0.959689 \\
\hline $\mathrm{H}$ & 3.35923100 & 4.07593300 & -0.815812 \\
\hline $\mathrm{C}$ & -3.22012600 & 3.34976100 & -0.5855880 \\
\hline $\mathrm{H}$ & -2.18783200 & 3.37905600 & -0.927819 \\
\hline $\mathrm{H}$ & -3.89395400 & 3.40347300 & -1.444020 \\
\hline $\mathrm{H}$ & -3.41245000 & 4.19727600 & 0.0759000 \\
\hline $\mathrm{H}$ & 0.86937100 & 0.22446900 & -3.702361 \\
\hline 0 & 0.80758100 & 0.35742400 & -2.743810 \\
\hline $\mathrm{H}$ & 0.65045500 & 1.30716200 & -2.632276 \\
\hline O & -0.09816000 & 0.57371400 & 2.783933 \\
\hline $\mathrm{H}$ & 0.19912800 & 1.48297100 & 2.62810 \\
\hline $\mathrm{H}$ & -0.15406300 & 0.48279300 & 3.74769 \\
\hline
\end{tabular}

$\begin{array}{crrr}\mathrm{Cu}\left[\left(\mathrm{CH}_{3} \mathrm{NHCOCH}_{2} \mathrm{CH}_{2}\right)_{2} \mathrm{NCH}_{2} \mathrm{CH}_{2} \mathrm{~N}\left(\mathrm{CH}_{2} \mathrm{CH}_{2} \mathrm{CONHCH}_{3}\right)_{2}\right]^{2+}(\mathbf{3 1}), & \\ \mathrm{E}_{\mathrm{e}}=-2977.2267637, \mathrm{ZPE}= & 0.5685889 & & \\ \mathrm{C} & -0.16956000 & -0.74549700 & -3.01621900 \\ \mathrm{H} & 0.73188900 & -1.35988600 & -3.00042000 \\ \mathrm{H} & -0.71113000 & -0.99012200 & -3.93878000 \\ \mathrm{C} & 0.19375500 & 0.73957000 & -3.01650700 \\ \mathrm{H} & -0.70773000 & 1.35399600 & -3.00762600 \\ \mathrm{H} & 0.74137100 & 0.98243400 & -3.93595300 \\ \mathrm{~N} & 1.00841000 & 1.10505100 & -1.81268400 \\ \mathrm{~N} & -0.99200700 & -1.10862900 & -1.81701200 \\ \mathrm{C} & 2.42822400 & 0.66257300 & -2.05455700 \\ \mathrm{H} & 2.40579200 & -0.35385100 & -2.44500500 \\ \mathrm{H} & 2.83816900 & 1.30458000 & -2.84408500\end{array}$




\begin{tabular}{|c|c|c|c|}
\hline $\mathrm{C}$ & 1.00447500 & 2.59930700 & -1.60096800 \\
\hline $\mathrm{H}$ & 2.02976600 & 2.91748100 & -1.40989000 \\
\hline $\mathrm{H}$ & 0.70132000 & 3.08460200 & -2.53377600 \\
\hline $\mathrm{C}$ & 3.36571700 & 0.69613900 & -0.84321200 \\
\hline $\mathrm{H}$ & 3.22837500 & 1.59912500 & -0.24127300 \\
\hline $\mathrm{H}$ & 4.39492300 & 0.72940900 & -1.21465600 \\
\hline $\mathrm{C}$ & 0.10425000 & 3.11446000 & -0.46706300 \\
\hline $\mathrm{H}$ & 0.14946500 & 4.20708000 & -0.47873700 \\
\hline $\mathrm{H}$ & -0.93631600 & 2.82328800 & -0.62478900 \\
\hline $\mathrm{C}$ & 3.17905300 & -0.51374400 & 0.06478300 \\
\hline 0 & 2.11260900 & -1.16511800 & 0.03928600 \\
\hline $\mathrm{N}$ & 4.20390700 & -0.82115200 & 0.87900400 \\
\hline $\mathrm{H}$ & 5.06002400 & -0.28873600 & 0.79311800 \\
\hline $\mathrm{C}$ & 4.18047100 & -1.93687600 & 1.82825000 \\
\hline $\mathrm{H}$ & 4.53611700 & -2.86031200 & 1.36135600 \\
\hline $\mathrm{H}$ & 3.15892300 & -2.08362600 & 2.17868500 \\
\hline $\mathrm{C}$ & 0.50687800 & 2.56716100 & 0.89009200 \\
\hline 0 & 0.66046300 & 1.32446800 & 1.06913800 \\
\hline $\mathrm{N}$ & 0.70155500 & 3.43918500 & 1.88581400 \\
\hline $\mathrm{H}$ & 0.58170600 & 4.42231700 & 1.67653900 \\
\hline $\mathrm{C}$ & 1.04481800 & 3.06945800 & 3.26403600 \\
\hline $\mathrm{H}$ & 1.06160400 & 1.98381200 & 3.33817200 \\
\hline $\mathrm{H}$ & 2.02662800 & 3.47054200 & 3.52783400 \\
\hline C & -2.41005900 & -0.66618100 & -2.06930000 \\
\hline $\mathrm{H}$ & -2.38481100 & 0.35022200 & -2.45955500 \\
\hline $\mathrm{H}$ & -2.81411300 & -1.30837000 & -2.86174900 \\
\hline $\mathrm{C}$ & -0.98982100 & -2.60245800 & -1.60241700 \\
\hline $\mathrm{H}$ & -2.01707800 & -2.92088900 & -1.42260900 \\
\hline $\mathrm{H}$ & -0.67574600 & -3.08952700 & -2.53064700 \\
\hline $\mathrm{C}$ & -0.10212900 & -3.11509300 & -0.45735300 \\
\hline $\mathrm{H}$ & 0.94046500 & -2.82632700 & -0.60581400 \\
\hline $\mathrm{H}$ & -0.14902200 & -4.20768400 & -0.46534100 \\
\hline C & -3.35692500 & -0.69928000 & -0.86541100 \\
\hline $\mathrm{H}$ & -4.38303200 & -0.73224700 & -1.24548100 \\
\hline $\mathrm{H}$ & -3.22508300 & -1.60227600 & -0.26231500 \\
\hline C & -0.51711300 & -2.56215100 & 0.89363100 \\
\hline O & -0.67025600 & -1.31848800 & 1.06731600 \\
\hline $\mathrm{N}$ & -0.72130100 & -3.42885700 & 1.89189300 \\
\hline $\mathrm{H}$ & -0.60722300 & -4.41375500 & 1.68808300 \\
\hline $\mathrm{C}$ & -1.08194800 & -3.04898900 & 3.26301400 \\
\hline $\mathrm{H}$ & -0.97495400 & -1.97055500 & 3.36459000 \\
\hline $\mathrm{H}$ & -0.41768400 & -3.55341700 & 3.96784300 \\
\hline C & -3.17832000 & 0.51132500 & 0.04352000 \\
\hline O & -2.11575200 & 1.16862600 & 0.01982000 \\
\hline $\mathrm{N}$ & -4.20667000 & 0.81154900 & 0.85635100 \\
\hline $\mathrm{H}$ & -5.05913800 & 0.27362700 & 0.76825700 \\
\hline $\mathrm{C}$ & 9299500 & 1.92652700 & 1 \\
\hline
\end{tabular}




$\begin{array}{lrrr}\mathrm{H} & -3.16918600 & 2.09489600 & 2.13987100 \\ \mathrm{H} & -4.81654000 & 1.67133900 & 2.66476300 \\ \mathrm{Cu} & 0.00432700 & -0.00084300 & -0.29333200 \\ \mathrm{H} & -4.57613700 & 2.84228200 & 1.34627200 \\ \mathrm{H} & 0.29785000 & 3.47257500 & 3.95187100 \\ \mathrm{H} & -2.11485700 & -3.33497600 & 3.47879500 \\ \mathrm{H} & 4.82351200 & -1.69603700 & 2.67608500\end{array}$

\section{$\mathrm{Cu}(\mathrm{OH})\left(\mathrm{H}_{2} \mathrm{O}\right)_{5}^{+}(\mathbf{3 2}), \mathrm{E}_{\mathrm{e}}=-2098.2229266, \mathrm{ZPE}=0.140243$}

$\begin{array}{lrrr}\mathrm{CU} & 0.420414 & 0.113807 & -0.216487 \\ \mathrm{O} & 1.562189 & -1.608144 & -0.051362 \\ \mathrm{H} & 1.043694 & -2.196102 & 0.527582 \\ \mathrm{O} & -0.523256 & -0.894984 & 1.644624 \\ \mathrm{H} & -0.339716 & -0.627462 & 2.557800 \\ \mathrm{O} & 2.110553 & 1.211688 & 0.214865 \\ \mathrm{H} & 1.862897 & 2.064946 & 0.608858 \\ \mathrm{O} & -0.586606 & 1.685403 & -0.078342 \\ \mathrm{H} & -0.532667 & 2.268623 & -0.852684 \\ \mathrm{O} & -2.713415 & 0.136813 & 0.295196 \\ \mathrm{H} & -3.651180 & 0.355311 & 0.396351 \\ \mathrm{O} & -0.946127 & -0.883903 & -1.419752 \\ \mathrm{H} & -1.006334 & -0.667229 & -2.363282 \\ \mathrm{H} & -1.478785 & -0.723541 & 1.495765 \\ \mathrm{H} & 1.810890 & -2.127579 & -0.832746 \\ \mathrm{H} & 2.879486 & 0.859461 & 0.690026 \\ \mathrm{H} & -1.823535 & -0.657854 & -1.011677 \\ \mathrm{H} & -2.183450 & 0.976030 & 0.220292\end{array}$

$\mathrm{Cu}(\mathrm{OH})\left(\mathrm{H}_{2} \mathrm{O}\right)_{2}\left[\left(\mathrm{CH}_{3}\right)_{2} \mathrm{NCH}_{2} \mathrm{CH}_{2} \mathrm{~N}\left(\mathrm{CH}_{3}\right)_{2}\right]^{+}(\mathbf{3 3}), \mathrm{E}_{\mathrm{e}}=-2216.70778092, \mathrm{ZPE}=0.291714$

$\begin{array}{lrrr}\mathrm{C} & 1.820735 & 1.262747 & 0.751536 \\ \mathrm{C} & 2.427587 & 0.038185 & 0.072904 \\ \mathrm{H} & 1.650469 & 1.064523 & 1.811823 \\ \mathrm{H} & 2.507721 & 2.115098 & 0.687514 \\ \mathrm{H} & 3.345461 & -0.266244 & 0.590779 \\ \mathrm{H} & 2.699752 & 0.266449 & -0.960202 \\ \mathrm{CU} & -0.419417 & -0.202070 & -0.284810 \\ \mathrm{O} & -1.293558 & -1.873763 & -0.249252 \\ \mathrm{H} & -1.535655 & -2.234250 & -1.115377 \\ \mathrm{O} & -3.343456 & -0.651226 & 0.913081 \\ \mathrm{H} & -2.752294 & -1.392361 & 0.613239 \\ \mathrm{O} & -2.174897 & 0.822527 & -0.845030 \\ \mathrm{H} & -2.496593 & 0.652531 & -1.743829 \\ \mathrm{~N} & 1.441012 & -1.077356 & 0.050573 \\ \mathrm{~N} & 0.502222 & 1.602568 & 0.134973 \\ \mathrm{H} & -2.842871 & 0.387880 & -0.224217\end{array}$




$\begin{array}{rrrr}\mathrm{H} & -4.208738 & -1.002568 & 1.161925 \\ \mathrm{C} & 0.684546 & 2.366318 & -1.131319 \\ \mathrm{H} & 1.281863 & 1.793250 & -1.843192 \\ \mathrm{H} & -0.293377 & 2.567234 & -1.569689 \\ \mathrm{H} & 1.189393 & 3.319585 & -0.933962 \\ \mathrm{C} & -0.309891 & 2.419086 & 1.082008 \\ \mathrm{H} & 0.218220 & 3.345280 & 1.339190 \\ \mathrm{H} & -1.263456 & 2.664699 & 0.615641 \\ \mathrm{H} & -0.496601 & 1.850080 & 1.995301 \\ \mathrm{C} & 1.357506 & -1.779625 & 1.361966 \\ \mathrm{H} & 1.140672 & -1.070925 & 2.163075 \\ \mathrm{H} & 0.549747 & -2.509432 & 1.309548 \\ \mathrm{H} & 2.306937 & -2.282991 & 1.580834 \\ \mathrm{C} & 1.770588 & -2.063333 & -1.011466 \\ \mathrm{H} & 2.756061 & -2.509055 & -0.827640 \\ \mathrm{H} & 1.011412 & -2.844729 & -1.007602 \\ \mathrm{H} & 1.781185 & -1.571084 & -1.986648\end{array}$

\section{$\mathrm{Cu}(\mathrm{OH})\left(\mathrm{H}_{2} \mathrm{O}\right)_{2}\left(\mathrm{H}_{2} \mathrm{NCH}_{2} \mathrm{CH}_{2} \mathrm{NHCOCH}_{3}\right)^{+}(\mathbf{3 4}), \mathrm{E}_{\mathrm{e}}=-2212.1402796, \mathrm{ZPE}=0.217725$}

$\begin{array}{lrrr}\mathrm{CU} & -0.757643 & -0.029156 & -0.153136 \\ \mathrm{~N} & -0.076524 & 1.804506 & 0.452803 \\ \mathrm{H} & -0.466938 & 2.456954 & -0.229387 \\ \mathrm{C} & 1.378143 & 2.077685 & 0.644572 \\ \mathrm{H} & 1.685888 & 1.646587 & 1.598763 \\ \mathrm{H} & 1.541741 & 3.157998 & 0.701039 \\ \mathrm{H} & -0.573809 & 2.019121 & 1.319855 \\ \mathrm{C} & 2.235981 & 1.491986 & -0.495977 \\ \mathrm{O} & 0.957757 & -1.036389 & 0.076249 \\ \mathrm{H} & 1.635758 & 1.397889 & -1.408778 \\ \mathrm{H} & 3.050974 & 2.180340 & -0.723829 \\ \mathrm{~N} & 2.864743 & 0.203365 & -0.185400 \\ \mathrm{H} & 3.875191 & 0.163926 & -0.196123 \\ \mathrm{C} & 2.212478 & -0.948617 & 0.053573 \\ \mathrm{C} & 3.036127 & -2.186792 & 0.305153 \\ \mathrm{H} & 2.811090 & -2.918193 & -0.475997 \\ \mathrm{H} & 4.110115 & -1.992217 & 0.317229 \\ \mathrm{H} & 2.733815 & -2.622880 & 1.260389 \\ \mathrm{O} & -1.693589 & -1.587057 & -0.499208 \\ \mathrm{O} & -2.503568 & 1.018112 & -0.656276 \\ \mathrm{H} & -3.187647 & 0.525503 & -0.093239 \\ \mathrm{O} & -3.784344 & -0.637821 & 0.842444 \\ \mathrm{H} & -3.199130 & -1.339946 & 0.462851 \\ \mathrm{H} & -2.742404 & 0.796314 & -1.571568 \\ \mathrm{H} & -4.688265 & -0.975959 & 0.900872 \\ \mathrm{H} & -1.118686 & -2.365337 & -0.442542\end{array}$




\section{$\mathrm{Cu}(\mathrm{OH})\left[\left(\mathrm{CH}_{3}\right)_{2} \mathrm{NCH}_{2} \mathrm{CH}_{2} \mathrm{~N}\left(\mathrm{CH}_{3}\right)_{2}\right]_{2}{ }^{+}(\mathbf{3 5}), \mathrm{E}_{\mathrm{e}}=-2411.6146859, \mathrm{ZPE}=0.467411$}

\begin{tabular}{|c|c|c|c|}
\hline CU & 0.180581 & 0.108780 & -0.471318 \\
\hline $\mathrm{N}$ & 1.213927 & 1.445439 & 0.939700 \\
\hline $\mathrm{N}$ & -1.828400 & 1.130413 & -0.677988 \\
\hline $\mathrm{N}$ & -1.486770 & -1.532876 & 0.948554 \\
\hline $\mathrm{N}$ & 2.128568 & -0.913415 & -0.503463 \\
\hline $\mathrm{C}$ & -2.785665 & -0.013832 & -0.586357 \\
\hline $\mathrm{H}$ & -3.806036 & 0.369726 & -0.730366 \\
\hline $\mathrm{H}$ & -2.552621 & -0.683861 & -1.412382 \\
\hline C & -2.732428 & -0.761196 & 0.746856 \\
\hline $\mathrm{H}$ & -3.609793 & -1.426912 & 0.794142 \\
\hline $\mathrm{H}$ & -2.839493 & -0.061339 & 1.579644 \\
\hline $\mathrm{C}$ & 2.659630 & 1.081344 & 0.849809 \\
\hline $\mathrm{H}$ & 3.194141 & 1.437436 & 1.740041 \\
\hline $\mathrm{H}$ & 3.085193 & 1.607787 & -0.006394 \\
\hline C & 2.853290 & -0.416297 & 0.691522 \\
\hline $\mathrm{H}$ & 3.925636 & -0.646348 & 0.620897 \\
\hline $\mathrm{H}$ & 2.467048 & -0.948430 & 1.564353 \\
\hline C & 2.060013 & -2.392519 & -0.443392 \\
\hline $\mathrm{H}$ & 1.524640 & -2.781885 & -1.309902 \\
\hline $\mathrm{H}$ & 3.068231 & -2.828638 & -0.436963 \\
\hline $\mathrm{H}$ & 1.536212 & -2.701705 & 0.461842 \\
\hline C & 2.827134 & -0.512914 & -1.754765 \\
\hline $\mathrm{H}$ & 3.842041 & -0.931440 & -1.779715 \\
\hline $\mathrm{H}$ & 2.262217 & -0.869005 & -2.615082 \\
\hline $\mathrm{H}$ & 2.888861 & 0.572776 & -1.832342 \\
\hline C & 1.105645 & 2.894202 & 0.617830 \\
\hline $\mathrm{H}$ & 0.103440 & 3.251745 & 0.838227 \\
\hline $\mathrm{H}$ & 1.815349 & 3.477645 & 1.218777 \\
\hline $\mathrm{H}$ & 1.321581 & 3.057207 & -0.440316 \\
\hline $\mathrm{C}$ & 0.736722 & 1.225254 & 2.330356 \\
\hline $\mathrm{H}$ & 1.294240 & 1.853800 & 3.037385 \\
\hline $\mathrm{H}$ & -0.321564 & 1.475681 & 2.399889 \\
\hline $\mathrm{H}$ & 0.860475 & 0.179764 & 2.612095 \\
\hline $\mathrm{C}$ & -1.341430 & -1.872200 & 2.375128 \\
\hline $\mathrm{H}$ & -2.173727 & -2.492967 & 2.744153 \\
\hline $\mathrm{H}$ & -0.413982 & -2.431063 & 2.532401 \\
\hline $\mathrm{H}$ & -1.305388 & -0.963069 & 2.980491 \\
\hline $\mathrm{C}$ & -1.543693 & -2.783351 & 0.164576 \\
\hline $\mathrm{H}$ & -0.648978 & -3.380079 & 0.355141 \\
\hline $\mathrm{H}$ & -2.420104 & -3.391659 & 0.439881 \\
\hline $\mathrm{H}$ & -1.584690 & -2.556880 & -0.900854 \\
\hline C & -2.259783 & 2.203543 & 0.248161 \\
\hline $\mathrm{H}$ & -2.120319 & 1.915432 & 1.290154 \\
\hline $\mathrm{H}$ & -1.696084 & 3.114144 & 0.049818 \\
\hline $\mathrm{H}$ & -3.323037 & 2.434517 & 0.096578 \\
\hline C & -1.878331 & 1.680889 & -2.062146 \\
\hline
\end{tabular}




$\begin{array}{rrrr}\mathrm{H} & -2.897210 & 2.011740 & -2.305627 \\ \mathrm{H} & -1.208449 & 2.541378 & -2.134531 \\ \mathrm{H} & -1.555141 & 0.912952 & -2.762002 \\ \mathrm{H} & 0.156858 & -1.523665 & -2.303352 \\ \mathrm{O} & -0.313038 & -0.706732 & -2.091369\end{array}$

$\mathrm{Cu}(\mathrm{OH})\left(\mathrm{H}_{2} \mathrm{NCH}_{2} \mathrm{CH}_{2} \mathrm{NHCOCH}_{3}\right)_{2}{ }^{+}(\mathbf{3 6}), \mathrm{E}_{\mathrm{e}}=-2402.5278343, \mathrm{ZPE}=0.318485$

$\begin{array}{lrrr}\mathrm{CU} & -0.010790 & -0.943240 & -0.244480 \\ \mathrm{~N} & -1.241290 & -1.148708 & 1.404766 \\ \mathrm{H} & -0.904784 & -1.911024 & 1.991928 \\ \mathrm{~N} & 1.044894 & -0.528626 & -1.965237 \\ \mathrm{H} & 0.347505 & 0.054811 & -2.429005 \\ \mathrm{H} & -1.058317 & -0.295981 & 1.933964 \\ \mathrm{H} & 1.010790 & -1.431773 & -2.439462 \\ \mathrm{C} & 2.377733 & 0.111594 & -2.053584 \\ \mathrm{H} & 2.538492 & 0.507977 & -3.062507 \\ \mathrm{H} & 3.143372 & -0.646897 & -1.875298 \\ \mathrm{C} & 2.528169 & 1.260664 & -1.037124 \\ \mathrm{H} & 3.164007 & 2.039098 & -1.462985 \\ \mathrm{H} & 1.547934 & 1.701625 & -0.833541 \\ \mathrm{~N} & 3.152494 & 0.873551 & 0.236135 \\ \mathrm{H} & 4.104190 & 1.173007 & 0.398036 \\ \mathrm{C} & 2.544355 & 0.209477 & 1.237185 \\ \mathrm{C} & 3.330414 & 0.004420 & 2.512223 \\ \mathrm{H} & 2.808733 & 0.511321 & 3.328779 \\ \mathrm{H} & 3.353738 & -1.062426 & 2.747359 \\ \mathrm{H} & 4.352712 & 0.383486 & 2.455070 \\ \mathrm{O} & 1.369302 & -0.224292 & 1.154163 \\ \mathrm{C} & -2.703549 & -1.305736 & 1.165933 \\ \mathrm{H} & -2.885231 & -2.338446 & 0.861430 \\ \mathrm{H} & -3.260309 & -1.120527 & 2.092321 \\ \mathrm{C} & -3.206552 & -0.399472 & 0.036898 \\ \mathrm{H} & -4.270000 & -0.600639 & -0.115945 \\ \mathrm{H} & -2.676730 & -0.657914 & -0.881996 \\ \mathrm{~N} & -3.058773 & 1.038143 & 0.298145 \\ \mathrm{H} & -3.867319 & 1.546312 & 0.629693 \\ \mathrm{C} & -1.985895 & 1.769700 & -0.102084 \\ \mathrm{C} & -2.098770 & 3.273955 & 0.029394 \\ \mathrm{H} & -1.207496 & 3.659581 & 0.529053 \\ \mathrm{H} & -2.127721 & 3.706637 & -0.975073 \\ \mathrm{H} & -2.988075 & 3.596065 & 0.575638 \\ \mathrm{O} & -0.952833 & 1.238317 & -0.561017 \\ \mathrm{O} & -0.890361 & -2.211304 & -1.344693 \\ \mathrm{H} & -1.018139 & -3.070231 & -0.914877\end{array}$

